\title{
The baryonic Tully-Fisher relation and its implication for dark matter halos ${ }^{\star}$
}

\author{
C. Trachternach ${ }^{1}$, W. J. G. de Blok ${ }^{2}$, S. S. McGaugh ${ }^{3}$, J. M. van der Hulst ${ }^{4}$, and R.-J. Dettmar ${ }^{1}$ \\ 1 Astronomisches Institut, Ruhr-Universität Bochum, Universitätsstraße 150, 44780 Bochum, Germany \\ 2 Department of Astronomy, University of Cape Town, Private Bag X3, Rondebosch 7701, South Africa \\ e-mail: edeblok@ast.uct.ac.za \\ 3 Department of Astronomy, University of Maryland, College Park, MD 20742-2421, USA \\ ${ }^{4}$ Kapteyn Astronomical Institute, University of Groningen, Postbus 800, 9700 AV Groningen, The Netherlands
}

Received 12 October 2008 / Accepted 28 July 2009

\section{ABSTRACT}

\begin{abstract}
Context. The baryonic Tully-Fisher relation (BTF) is a fundamental relation between baryonic mass and maximum rotation velocity. It can be used to estimate distances, as well as to constrain the properties of dark matter and its relation with the visible matter. Aims. In this paper, we explore if extremely low-mass dwarf galaxies follow the same BTF relation as high-mass galaxies. We quantify the scatter in the BTF relation and use this to constrain the allowed elongations of dark matter halo potentials.

Methods. We obtained H I synthesis data of 11 dwarf galaxies and derive several independent estimates for the maximum rotation velocity.

Results. Constructing a BTF relation using data from the literature for the high-mass end, and galaxies with detected rotation from our sample for the low-mass end results in a BTF with a scatter of $0.33 \mathrm{mag}$.

Conclusions. This scatter constrains the ellipticities of the potentials in the plane of the disks of the galaxies to an upper limit of $0-0.06$, indicating that dwarf galaxies are at most only mildly tri-axial. Our results indicate that the BTF relation is a fundamental relation which all rotationally dominated galaxies seem to follow.
\end{abstract}

Key words. dark matter - galaxies: kinematics and dynamics - galaxies: dwarf - galaxies: fundamental parameters

\section{Introduction}

The Tully-Fisher (TF) relation (Tully \& Fisher 1977), the relation between the luminosity and rotation velocity of a galaxy, has been extensively used to estimate extragalactic distances (e.g., Pierce \& Tully 1988; Willick et al. 1997; Sakai et al. 2000; Tully \& Pierce 2000; Springob et al. 2007). In the usual "classical" interpretation, luminosity is a proxy for the stellar mass, which in turn depends on the total (visible and dark) mass and through it on the rotation velocity. The slope and zero point of this classical TF relation do not depend on the central surface brightness of galaxies (Zwaan et al. 1995), though for very low mass dwarf galaxies, the slope tends to steepen (e.g., Matthews et al. 1998; McGaugh et al. 2000). Low-mass dwarf galaxies are apparently underluminous given their rotation velocity and therefore fall below the TF relation as defined by the high mass galaxies. A single linear relation can be restored if one replaces the luminosity (or stellar mass) with the baryonic disk mass, thus including the gas mass as well (McGaugh et al. 1999, 2000). This relation is called the baryonic Tully-Fisher (BTF) relation and has been studied by many authors in the last few years (e.g., Bell \& de Jong 2001; Verheijen 2001; Gurovich et al. 2004; McGaugh 2004, 2005; Geha et al. 2006; De Rijcke et al. 2007; Noordermeer \& Verheijen 2007; Stark et al. 2009).

The existence of a (baryonic) Tully-Fisher relation places severe constraints on galaxy formation and evolution theories

\footnotetext{
* Appendix is only available in electronic form at http://www . aanda . org
}

(cf. Eisenstein \& Loeb 1996; McGaugh \& de Blok 1998a,b; Mo et al. 1998; Steinmetz \& Navarro 1999; Blanton et al. 2008). For example, Franx \& de Zeeuw (1992) note that the observed scatter in the TF relation places upper limits on the elongation of dark matter halos. They find that that the ellipticity of the potential in the plane of the disk is most likely between 0 and $\sim 0.06$. This is in good agreement with what Trachternach et al. (2008) found observationally for a sample of 18 dwarf and spiral galaxies from the THINGS survey (Walter et al. 2008; de Blok et al. 2008), but in disagreement with results from CDM simulations which predict more elongated potentials (e.g., Frenk et al. 1988; Hayashi \& Navarro 2006). The smaller scatter in the observed BTF relation (e.g., McGaugh 2005), means that it can be used to put similar constraints on lower mass galaxies as well. This was recently shown by Stark et al. (2009) who studied the BTF for a large sample of dwarf galaxies with resolved $\mathrm{H}$ I rotation curves, and found a linear BTF relation with small scatter.

The low mass end of the BTF relation has also been studied by Geha et al. (2006) and Kovac (2007). They measure line widths and after correcting these for broadening due to turbulent motion of the $\mathrm{HI}$, they find that the extreme dwarf galaxies of their sample follow the same BTF relation as the high mass galaxies, albeit with a larger scatter. This increased scatter is most likely a result of their use of the $W_{20}$ profile. Broeils (1992) and Verheijen (1997) already pointed out that using the maximum rotation velocity from a resolved rotation curve significantly decreases the scatter as compared to using line width measurements. 
Table 1. Properties of the data.

\begin{tabular}{|c|c|c|c|c|c|c|c|c|}
\hline ID & $\begin{array}{c}\text { Date } \\
\text { of Obs. } \\
(2)\end{array}$ & $\begin{array}{l}\text { length } \\
\text { of Obs. } \\
\text { (3) }\end{array}$ & $\begin{array}{c}b_{\text {maj }} \\
" \\
(4)\end{array}$ & $\begin{array}{c}b_{\min } \\
\prime \prime \\
(5)\end{array}$ & $\begin{array}{l}\text { PA } \\
\circ \\
(6)\end{array}$ & $\begin{array}{c}\text { Noise/channel } \\
\text { mJy beam }^{-1} \\
\text { (7) }\end{array}$ & $\begin{array}{c}\text { Pixel size } \\
\text { (1 } \\
\text { (8) }\end{array}$ & $\begin{array}{c}\text { Channel width } \\
\mathrm{km} \mathrm{s}^{-1} \\
\text { (9) }\end{array}$ \\
\hline D500-2 & $01-05-2002$ & $12 \mathrm{~h}$ & 25.0 & 10.6 & 0.0 & 1.0 & 5 & 4.12 \\
\hline D500-3 & 03-05-2002 & $12 \mathrm{~h}$ & 33.0 & 12.3 & 0.0 & 0.6 & 5 & 4.12 \\
\hline D512-2 & $16-05-2004$ & $12 \mathrm{~h}$ & 28.9 & 13.4 & 0.6 & 0.9 & 4 & 2.10 \\
\hline D564-8 & 08-05-2002 & $12 \mathrm{~h}$ & 36.4 & 12.8 & 0.0 & 0.6 & 5 & 4.12 \\
\hline D572-5 & $10-05-2004$ & $12 \mathrm{~h}$ & 86.6 & 24.0 & -0.2 & 1.2 & 4 & 2.10 \\
\hline D575- $1^{a}$ & 07-05-2004 & $12 \mathrm{~h}$ & 48.2 & 18.4 & 0.2 & 0.9 & 4 & 2.10 \\
\hline$\ldots$ & $12-05-2004$ & $12 \mathrm{~h}$ & $\ldots$ & $\ldots$ & $\ldots$ & $\ldots$ & $\ldots$ & $\ldots$ \\
\hline D575- $2^{b}$ & 09-05-2004 & $12 \mathrm{~h}$ & 45.6 & 14.0 & -3.5 & 0.6 & 4 & 2.10 \\
\hline$\ldots$ & $13-05-2004$ & $10 \mathrm{~h}$ & $\ldots$ & $\ldots$ & $\ldots$ & $\ldots$ & $\ldots$ & $\ldots$ \\
\hline$\ldots$ & $17-10-2004$ & $7 \mathrm{~h}$ & $\ldots$ & $\ldots$ & $\ldots$ & $\ldots$ & ... & $\ldots$ \\
\hline D575-5 & $05-05-2004$ & $12 \mathrm{~h}$ & 37.5 & 11.3 & -0.1 & 1.1 & 4 & 2.10 \\
\hline D631-7 & 04-11-2004 & $12 \mathrm{~h}$ & 57.7 & 12.0 & 0.2 & 1.1 & 4 & 2.10 \\
\hline D640-13 & $10-11-2004$ & $12 \mathrm{~h}$ & 70.8 & 11.5 & -0.1 & 1.1 & 4 & 2.10 \\
\hline D646-7 & $11-05-2004$ & $12 \mathrm{~h}$ & 133.8 & 20.6 & 0.6 & 1.4 & 4 & 2.10 \\
\hline
\end{tabular}

${ }^{a}$ The data for D575-1 are combined from two individual observations.

${ }^{b}$ The data for D575-2 are combined from three individual observations.

Notes: (1) galaxy identifier; (2) date of observations; (3) length of observations; $(4,5)$ major and minor axis diameter of the robust weighted beam in arcsec; (6) position angle of the beam (in degrees), measured counter-clockwise from the north; (7) noise per channel in mJy beam ${ }^{-1}$; (8) pixel size in arcsec; (9) channel width in $\mathrm{km} \mathrm{s}^{-1}$.

In this paper, we attempt to determine several independent estimates for the maximum rotation velocity $\left(V_{\max }\right)$ for a sample of extremely low-mass dwarf galaxies. Doing this, we can check whether these galaxies follow the same tight correlation between baryonic disk mass and rotation velocity as their high mass counterparts. As a reference, we use the sample of McGaugh (2005), for which well-determined estimates for $V_{\max }$ exist based on analysis of well-resolved rotation curves. For an in-depth description of the comparison sample, the reader is referred to McGaugh (2005) and references therein.

The paper is organized as follows: in Sect. 2 we describe the observations and the data reduction, which is followed by a description of the different methods in estimating $V_{\max }$ in Sect. 3 . We comment the individual galaxies in Sect. 4, and present further analysis and our results in Sect. 5. We summarize our results and give our conclusions in Sect. 6. The Appendix contains moment maps, position-velocity diagrams and channel maps of our sample galaxies.

\section{The data}

Our sample was selected from the larger sample of Schombert et al. (1997), which is one of the largest samples of extreme field dwarf galaxies for which both line width measurements and H I masses (Eder \& Schombert 2000), as well as optical photometry (Pildis et al. 1997) exist. The galaxies were chosen to be relatively nearby $\left(v_{\text {hel }}<1400 \mathrm{~km} \mathrm{~s}^{-1}\right)$, have suitable optical inclinations for potential derivation of their rotation curves ( $45 \leq i_{\mathrm{opt}} \leq 75$ ), and to have $V$ - and $I$-band photometry available.

\subsection{Observations}

Observations were carried out at the Westerbork Synthesis Radio Telescope (WSRT) in maxi-short configuration, in the $21-\mathrm{cm}$ line of neutral hydrogen. We use both polarizations and sample 1024 channels with a bandwidth of $10 \mathrm{MHz}$ or $20 \mathrm{MHz}$ (corresponding to velocity resolutions of 2.10 or $4.12 \mathrm{~km} \mathrm{~s}^{-1}$ ). The sample integration time was set to $60 \mathrm{~s}$. Further observational details are summarized in Table 1.

\subsection{Data reduction}

The calibration and data reduction of the data is performed using standard routines in MIRIAD ${ }^{1}$. The data are calibrated using one of the standard primary calibrators used at the WSRT (3C48, 3C147, 3C286, J2052+362). The primary calibrators are also used for the bandpass and gain corrections.

The line-free channels (i.e., the channels containing only continuum emission) were used to create a continuum image. This image was used to self-calibrate the data. After the selfcalibration and continuum subtraction, image cubes were created using the robust weighting scheme (Briggs 1995) with a robust parameter of zero for all galaxies. These image cubes were then cleaned down to a level of $\sim 1 \sigma$. For a list of the beam sizes and noise levels, see Table 1 . We additionally created Hanning smoothed data cubes, which were used for the creation of the $\mathrm{HI}$ profiles and the derivation of the velocity widths.

Using GIPSY ${ }^{2}$, we created zeroth, first and second moment maps of all galaxies. In order to isolate significant signal, we smoothed the data cubes to half the original resolution and only retained pixels with values $>2.5$ times the (smoothed) noise value. Spurious pixels were blotted by hand. This smoothed and blotted data cube was used as a mask for the original data cube. We determined the number of channels with significant emission contributing to each unblanked pixel in the unsmoothed moment maps and created a map containing the signal-to-noise $(S / N)$ of each pixel in the total intensity (zeroth moment) map. Using this map, we determined the average pixel value in the zeroth moment map corresponding to a $S / N=3$, and clip all moment maps using this flux limit. Channel maps and moment maps are presented in the Appendix.

\footnotetext{
1 Multichannel Image Reconstruction, Image Analysis and Display (Sault et al. 1995).

2 GIPSY, the Groningen Image Processing SYstem (van der Hulst et al. 1992).
} 


\section{Estimating the maximum rotation velocity}

In order to construct a (baryonic) Tully-Fisher relation, one needs to estimate the maximum (outer) rotation velocity $\left(V_{\max }\right)$ of a galaxy. There are several ways in which this can be done, which are described below in increasing order of preference.

\subsection{HI velocity profile}

The simplest way is to use the width $W$ of the global H I velocity profile, usually measured at the 20 (50) percent level of the maximum intensity, and denoted as $W_{20}\left(W_{50}\right)$. The advantage in using the profile width is that it is easy to measure and can be derived using low resolution data. The drawback is that one cannot distinguish between rotation and turbulence. This uncertainty matters little for large, fast rotating spiral galaxies. However, as one goes to smaller and more slowly rotating galaxies, turbulent motions will start to contribute significantly to the total width of H I profiles (cf. Verheijen 1997). In our analysis, we use the velocity profiles from the Hanning smoothed data cubes and correct them for instrumental broadening and turbulent motion. The corrections applied are addressed and discussed more fully in Sect. 5.2.

\subsection{Major axis position-velocity diagram}

An alternative way to derive $V_{\max }$ is to make use of the major axis position-velocity $(p V)$ diagram. For a galaxy with a flat rotation curve, Gaussian fits to the outer galaxy radii in the major axis $p V$-diagram (see, e.g., Fig. A. 1 in the Appendix) yield an estimate for the (projected) amplitude of the rotation. The maximum rotation velocity is then calculated as half the difference in velocity between approaching and receding sides, corrected for inclination:

$V_{p v}=\frac{V_{\text {receding }}-V_{\text {approaching }}}{2 \sin (i)}$.

The $p V$-diagram is potentially able to better constrain the the maximum rotation curve velocity than $W_{20}$ and $W_{50}$ values (Verheijen 2001).

\subsection{Tilted-ring models}

A third method for the derivation of $V_{\max }$ is a tilted-ring model, in which the kinematics of a galaxy are described using a set of concentric rings. Each of these rings can have its own center position $\left(x_{0}, y_{0}\right)$, systemic velocity $V_{\text {sys }}$, rotation velocity $V_{\text {rot }}$, inclination $i$, and position angle PA. The pre-requisite for this method is a resolved velocity field showing signs of rotation.

For the galaxies in our sample that meet this criterion, we derive rotation curves using the GIPSY task ROTCUR. Assuming that the gas moves on circular orbits, the line-of-sight velocity can be expressed as:

$V(x, y)=V_{\text {sys }}+V_{\text {rot }}(r) \sin (i) \cos (\theta)$.

Here, $\theta$ is the azimuthal distance from the major axis in the plane of the galaxy and is related to the position angle PA of the galaxy as measured in the plane of the sky by

$$
\cos (\theta)=\frac{-\left(x-x_{0}\right) \sin (\mathrm{PA})+\left(y-y_{0}\right) \cos (\mathrm{PA})}{r}
$$

and

$$
\sin (\theta)=\frac{-\left(x-x_{0}\right) \cos (\mathrm{PA})-\left(y-y_{0}\right) \sin (\mathrm{PA})}{r \cos (i)} .
$$

The PA is measured counter-clockwise from the north to the major axis of the receding side of the galaxy. As positions along the major axis of a galaxy carry more rotational information than positions near the minor axis, we weight the individual data points by $|\cos (\theta)|$.

The derivation of a rotation curve is generally an iterative process involving the consecutive determination of the various tilted-ring parameters. Following is a general description of the procedure applied. In order to get good initial estimates for $i$, $\mathrm{PA}$, and the center position, we fit isophotes at varying intensity levels to the H I total intensity maps, taking care that the results are not affected by small-scale structures. As initial estimates for $V_{\text {sys }}$ and $V_{\text {rot }}$, we use the central velocities of the $W_{50}$ profile and $\frac{1}{2} W_{50} / \sin (i)$, respectively.

In the first tilted-ring fit, we determine the systemic velocity by keeping all parameters except $V_{\text {sys }}$ and $V_{\text {rot }}$ fixed. In a second run, we derive the position of the dynamical center leaving by leaving (only) the central position of the rings and $V_{\text {rot }}$ unconstrained. In a third fit, we determine the position angle by leaving only PA, $i$ and $V_{\text {rot }}$ as free parameters. As in most cases we sample the rotation curves with relatively few $(\sim 10)$ tilted-rings, we approximate the fitted values either by a constant or linearly changing PA. Once the PA is modeled in such a way, we make another fit with only $i$ (and $V_{\text {rot }}$ ) as free parameters and derive the inclination of the galaxy. We do not model any radial trends for the inclination. In a last run, we determine the rotation curve by keeping all parameters except $V_{\text {rot }}$ fixed at their best determined values. The maximum velocity of the rotation curve derived in this way will be referred to as $V_{v f}$ hereafter.

\section{Comments on individual galaxies}

In this section, we present our results for the individual galaxies. Unless mentioned otherwise, the distances given in the following sections are calculated by correcting the systemic velocities of the galaxies for Virgocentric infall (following the formalism presented in Mould et al. 2000), and assuming a Hubble flow with a Hubble constant of $H_{0}=75 \mathrm{~km} \mathrm{~s}^{-1} \mathrm{Mpc}^{-1}$. Given the different distance outcomes of different flow models, we use half the difference between the minimum and maximum flow-corrected distances reported in NED as an indication of the uncertainty in the distance. Where independent distance measurements through measurements of the luminosity of the tip of the red giant branch are available, we adopt the distances and uncertainties listed in the source paper.

\subsection{Sub-samples}

As will be discussed extensively in Sect. 5.4, we will split our sample into two sub-samples, namely the "profile width" subsample and the "rotation curve" sub-sample. The profile width sub-sample contains the galaxies for which it proved impossible to derive a maximum rotation velocity from either the tiltedring analysis $\left(V_{v f}\right)$ or from the position-velocity diagram $\left(V_{p v}\right)$. For this sample, therefore, only $W_{20}$ and $W_{50}$ measurements are available. Their properties are summarized in Table 2. The rotation curve sub-sample is summarized in Table 3. It contains the galaxies for which we were able to derive $V_{v f}$ and/or $V_{p v}$ (in addition to $W_{20}$ and $W_{50}$ ).

In the Appendix, we show for all galaxies presented in this paper a summary panel consisting of moment maps, major and minor axis position-velocity diagrams and the global $\mathrm{H}$ I profile. 
Table 2. Derived parameters for the profile width sub-sample.

\begin{tabular}{|c|c|c|c|c|c|c|c|c|c|c|c|c|}
\hline (1) & $\begin{array}{c}\alpha_{2000} \\
(\mathrm{~h} \mathrm{~m} \mathrm{~s}) \\
(2)\end{array}$ & $\begin{array}{c}\delta_{2000} \\
\left(\circ^{\prime} \prime \prime\right) \\
(3)\end{array}$ & $\begin{array}{c}V_{\text {sys }} \\
\mathrm{km} \mathrm{s}^{-1} \\
(4)\end{array}$ & $\begin{array}{c}\mathrm{D} \\
\mathrm{Mpc} \\
(5)\end{array}$ & $\begin{array}{c}M_{V} \\
\text { mag } \\
(6)\end{array}$ & $\begin{array}{c}(V-I) \\
(7) \\
\end{array}$ & $\begin{array}{c}M_{\mathrm{HI}} \\
10^{7} M_{\odot} \\
(8)\end{array}$ & $\begin{array}{c}W_{20} \\
\mathrm{~km} \mathrm{~s}^{-1} \\
(9)\end{array}$ & $\begin{array}{c}W_{50} \\
\mathrm{~km} \mathrm{~s}^{-1} \\
(10)\end{array}$ & $\begin{array}{c}W_{20, \text { turb }} \\
\mathrm{km} \mathrm{s}^{-1} \\
(11)\end{array}$ & $\begin{array}{c}W_{50, \text { turb }} \\
\mathrm{km} \mathrm{s}^{-1} \\
(12)\end{array}$ & $\begin{array}{c}i \\
\circ \\
(13) \\
\end{array}$ \\
\hline D572-5 & 114816.4 & +183833 & 994 & 14.6 & -14.56 & 0.52 & 8.55 & 77 & 62 & 68 & 55 & $50^{a}$ \\
\hline D575-1 & 125146.1 & +214407 & 600 & 10.0 & -14.22 & 0.70 & 3.76 & 38 & 24 & 30 & 18 & 53 \\
\hline D575-5 & 125541.4 & +191234 & 437 & 7.7 & -13.21 & 0.44 & 4.27 & 28 & 19 & 16 & 10 & 50 \\
\hline D640-13 & 105613.9 & +120041 & 958 & 13.4 & -14.36 & 0.55 & 4.53 & 38 & 25 & 30 & 18 & $48^{a}$ \\
\hline D646-7 & 125840.4 & +141303 & 233 & $2.1^{b}$ & -12.52 & 0.84 & 0.37 & 35 & 23 & 26 & 16 & $55^{a}$ \\
\hline
\end{tabular}

${ }^{a}$ Optical $I$-band inclination from Pildis et al. (1997).

${ }^{b}$ Distance as given in Karachentsev et al. (2003).

Notes: (1) galaxy identifier; $(2,3)$ central position taken from NED; (4) systemic velocity derived from the central velocity of the $W_{50}$ profile; (5) distance in Mpc. If no reference is given, the distance is based on $V_{\text {sys }}$ (Col. 4) and a Hubble flow using $H_{0}=75 \mathrm{~km} \mathrm{~s}^{-1} \mathrm{Mpc}^{-1}$, including a correction for Virgocentric infall (Mould et al. 2000); (6) absolute $V$-band magnitude as given in Pildis et al. (1997), corrected to our distance estimates; (7) $V-I$ color from Pildis et al. (1997); (8) total H I mass (in units of $10^{7} M_{\odot}$ ); (9) uncorrected width of the H I profile at the 20 percent level of the maximum intensity. The values listed here for $W_{20}$ are derived using the Hanning smoothed data; (10) as Col. 9, but for the $W_{50}$ profile; (11) width of the $W_{20}$ profile, corrected for the finite velocity resolution, and turbulent motion of the $\mathrm{H}$ I gas; (12) as Col. 11, but for the $W_{50}$ profile; (13) adopted inclination angle. The velocity widths in Cols. (9)-(12) are not corrected for inclination effects.

Table 3. Derived parameters for the rotation curve sub-sample.

\begin{tabular}{|c|c|c|c|c|c|c|c|c|c|c|c|c|c|c|c|}
\hline (1) & $\begin{array}{c}\alpha_{2000} \\
(\mathrm{~h} \mathrm{~m} \mathrm{~s}) \\
(2)\end{array}$ & $\begin{array}{c}\delta_{2000} \\
\left(0^{\prime} \prime \prime\right) \\
(3)\end{array}$ & $\begin{array}{c}V_{\text {sys }} \\
\mathrm{km} \mathrm{s}^{-1} \\
(4)\end{array}$ & $\begin{array}{c}\mathrm{D} \\
\mathrm{Mpc} \\
(5)\end{array}$ & $\begin{array}{c}M_{V} \\
\text { mag } \\
(6)\end{array}$ & $\overline{(\overline{(V-I)}}$ & $\begin{array}{c}M_{\mathrm{HI}} \\
10^{7} M_{\odot} \\
(8) \\
\end{array}$ & $\begin{array}{c}W_{20} \\
\mathrm{~km} \mathrm{~s}^{-1} \\
(9)\end{array}$ & $\begin{array}{c}W_{50} \\
\mathrm{~km} \mathrm{~s}^{-1} \\
(10)\end{array}$ & $\begin{array}{c}W_{20,} \text { turb } \\
\mathrm{km} \mathrm{s}^{-1} \\
(11)\end{array}$ & $\begin{array}{c}W_{50, \text { turb }} \\
\mathrm{km} \mathrm{s}^{-1} \\
(12)\end{array}$ & $\begin{array}{c}i \\
\circ \\
(13) \\
\end{array}$ & $\begin{array}{c}\langle\mathrm{PA}\rangle \\
\circ \\
(14)\end{array}$ & $\begin{array}{c}V_{v f} \\
\mathrm{~km} \mathrm{~s}^{-1} \\
(15)\end{array}$ & $\begin{array}{c}V_{p v} \\
\mathrm{~km} \mathrm{~s}^{-1} \\
(16)\end{array}$ \\
\hline D500-2* & 103143.0 & +251833 & 1259 & 17.9 & -16.38 & 0.42 & 87.08 & 135 & 120 & 119 & 105 & $57 \pm 6$ & 345 & 68 & 68 \\
\hline D500-3 & 100559.5 & +235204 & 1327 & 18.5 & -15.74 & 0.31 & 6.58 & 96 & 83 & 84 & 72 & $55^{a} \pm 6$ & .. & $\ldots$ & 45 \\
\hline D512-2 & 143320.2 & +265954 & 840 & 14.1 & -15.22 & 0.80 & 6.96 & 88 & 74 & 78 & 65 & $56 \pm 10$ & 40 & 35 & 37 \\
\hline D564-8* & 090254.0 & +200428 & 478 & 6.5 & -12.64 & 0.93 & 1.58 & 62 & 48 & 54 & 42 & $63 \pm 7$ & 13 & 25 & 29 \\
\hline D575-2* & 125221.9 & +213746 & 774 & 12.2 & -15.15 & 0.78 & 31.51 & 146 & 119 & 129 & 104 & $57 \pm 5$ & 220 & 74 & .. \\
\hline D631-7* & 075701.8 & +142327 & 311 & $5.5^{b}$ & -14.50 & 0.55 & 14.68 & 113 & 89 & 100 & 78 & $59 \pm 3$ & 324 & 58 & 53 \\
\hline
\end{tabular}

${ }^{a}$ Optical $I$-band inclination from Pildis et al. (1997).

${ }^{b}$ Distance as given in Karachentsev et al. (2003).

Notes: Cols. (1) to (12) as in Table 2, except that for the starred galaxies, the center position and $V_{\text {sys }}$ were derived kinematically; (13) inclination angle (optical inclination for D500-3, kinematic inclination otherwise). The uncertainties given for the kinematic inclination angles represent the scatter of the values of the individual tilted-rings. For D500-3, the average uncertainty of the kinematic inclination estimates is given; (14) average position angle of the rotation curve analysis, measured counter-clockwise from north to the receding side of the galaxy; (15) maximum rotation velocity from the tilted-ring analysis of the velocity field, corrected for inclination; (16) maximum rotation velocity from the position-velocity diagram, corrected for inclination.

We additionally show channel maps for the galaxies of the rotation curve sub-sample.

\subsection{D500-2 (Data presented in Figs. 1, A.1, and A.12)}

D500-2 is also known as UGC 5716 and is classified as an Sm galaxy. We assume a distance of $17.9 \pm 3.4 \mathrm{Mpc}$. The global H I profile of D500-2 (cf. Fig. A.1) shows the double-horned profile typical for spiral galaxies, and its velocity field indicates that D500-2 is clearly dominated by rotation. We fit ellipses using the zeroth moment map at a few representative intensity levels. The resulting inclination of the H I disk, corrected for the beam size, is $i_{\mathrm{HI}} \sim 57^{\circ}$. The parameters from these isophote fits are used as initial estimates for the tilted-ring analysis. The width of the rings is set to $11^{\prime \prime}$. The systemic velocity as derived in the first tilted-ring fit shows only small radial variation. Its mean value is $V_{\text {sys }}=1259 \mathrm{~km} \mathrm{~s}^{-1}$, identical to the the central velocity of the $W_{50}$ profile. Fixing the systemic velocity, we derive the dynamical center in a new fit by averaging the $\left(x_{0}, y_{0}\right)$ values over the entire radial range. The resulting central position (cf. Table 3) is in good agreement with the optical center from NED and with that derived from our ellipse fits to the zeroth moment map. Keeping the center position fixed for the subsequent tilted-ring fits, we derive the PA and inclination angle. The position angle shows a linear decrease from $350^{\circ}$ in the center to $340^{\circ}$ in the outer parts.

The inclination is then determined in an additional fit by averaging the inclination values for $r \geq 35^{\prime \prime}$. The resulting kinematic inclination is $i_{\text {kin }} \sim 57^{\circ} \pm 6^{\circ}$, which agrees with the (beam corrected) inclination of the H I disk. In a last tilted-ring fit, we derive the rotation velocity by keeping all parameters except $V_{\text {rot }}$ fixed to their previously derived values. After a gentle inner rise, the rotation curve of D500-2 reaches a flat part at a velocity of $V_{v f} \sim 68 \mathrm{~km} \mathrm{~s}^{-1}$. The rotation curve, as well as the radial distributions for PA and $i_{\text {kin }}$ are shown in Fig. 1. The maximum rotation velocity from the $p V$-diagram is $V_{p v} \sim 68 \mathrm{~km} \mathrm{~s}^{-1}$.

\subsection{D500-3 (Data presented in Figs. A.2 and A.13)}

D500-3 is a dwarf irregular (dI) at a distance of $18.5 \pm 3.2 \mathrm{Mpc}$. Its velocity field (cf. Fig. A.2) indicates solid-body rotation throughout the entire disk, as confirmed by the $p V$-diagram. We fit isophotes to different intensity levels in the zeroth moment map and find an inclination of the $\mathrm{HI}$ disk $i_{\mathrm{HI}} \sim 42^{\circ}$. This value is somewhat smaller than the optical $I$-band inclination of $i_{\text {opt }} \sim 55^{\circ}$ as given in Pildis et al. (1997). Because of the large $\mathrm{H}$ I beam size, we consider the optical inclination superior to the $\mathrm{HI}$ inclination and use it in our further analysis. The solid-body rotation and the limited spatial resolution make it impossible to 


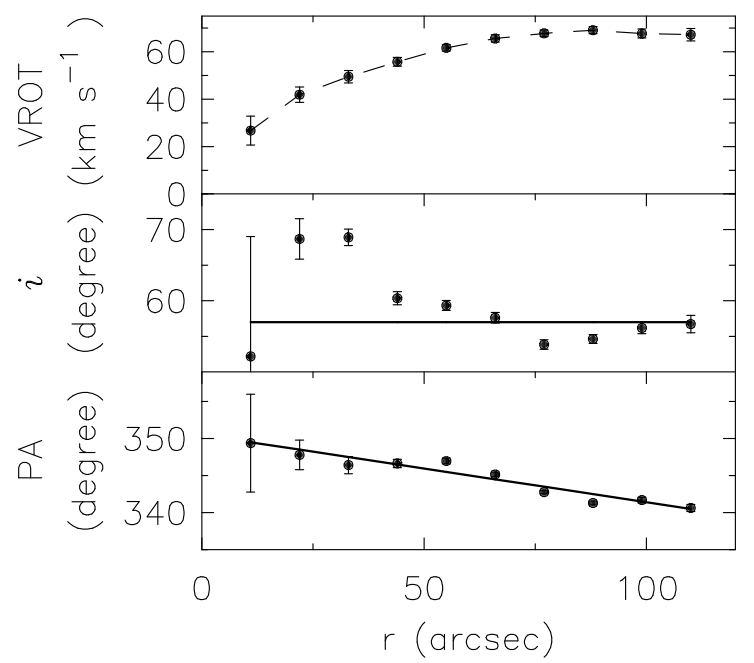

Fig. 1. Tilted-ring analysis of D500-2. From top to bottom, the radial distributions of the rotation velocity, the inclination angle, and the position angle are shown. The data points in the center and bottom panel indicate the values derived for $i$ and PA as free parameters. The solid lines indicate the values adopted to derive the rotation curve shown in the top panel.

derive a well-determined rotation curve using tilted-ring models. We are, however, able to get an estimate for the maximum rotation velocity by using the position-velocity diagram and find $V_{p v} \sim 45 \mathrm{~km} \mathrm{~s}^{-1}$.

\subsection{D512-2 (Data presented in Figs. 2, A.3, and A.14)}

D512-2 is a galaxy of Hubble type Sm. We assume a distance of $14.1 \pm 2.2 \mathrm{Mpc}$. The velocity field of D512-2 shows signs of solid-body rotation in its inner parts. We fit ellipses at a number of representative intensity levels in the zeroth moment map and derive an inclination of the $\mathrm{HI}$ disk of $i_{\mathrm{HI}} \sim 48^{\circ}$ (corrected for the beam size). Because the apparent size of D512-2 is small, we do not fit the center position or the systemic velocity using tilted-ring fits. For the center position, we adopt the estimate derived from the ellipse fitting, which is in good agreement with the position of the optical center. For the systemic velocity, we use the central velocity of the $W_{50}$ profile. Keeping the center and the systemic velocity fixed, we derive an average position angle $\mathrm{PA} \sim 40^{\circ}$ from the tilted ring fit, choosing the width of the tilted-rings to be $13^{\prime \prime}$. The position angle is then kept fixed for a subsequent tilted-ring fit to estimate $i$. Averaging the resulting inclination values yields $i_{\text {kin }} \sim 56^{\circ} \pm 10^{\circ}$, which is consistent with the values discussed above. Using the kinematic inclination, we determine the rotation curve in a last tilted-ring run leaving only $V_{\text {rot }}$ unconstrained. The rotation curve (cf. Fig. 2) confirms what was already suggested by the velocity field: a solid-body rotation in the inner parts and a flat part in the outer regions. The rotation velocity of the flat part of the rotation curve is $V_{v f} \sim 35 \mathrm{~km} \mathrm{~s}^{-1}$. From the $p V$-diagram, we estimate the (inclination corrected) maximum rotation velocity to be $V_{p v} \sim 37 \mathrm{~km} \mathrm{~s}^{-1}$.

\subsection{D564-8 (Data presented in Figs. 3, A.4, and A.15)}

For the dwarf irregular D564-8 (also known as F564-V3, see de Blok et al. 1996), we assume a distance of $6.5 \pm 2.6 \mathrm{Mpc}$. The global H I profile of D564-8 is asymmetric with its peak flux density towards the receding side of the galaxy. The asymmetry of

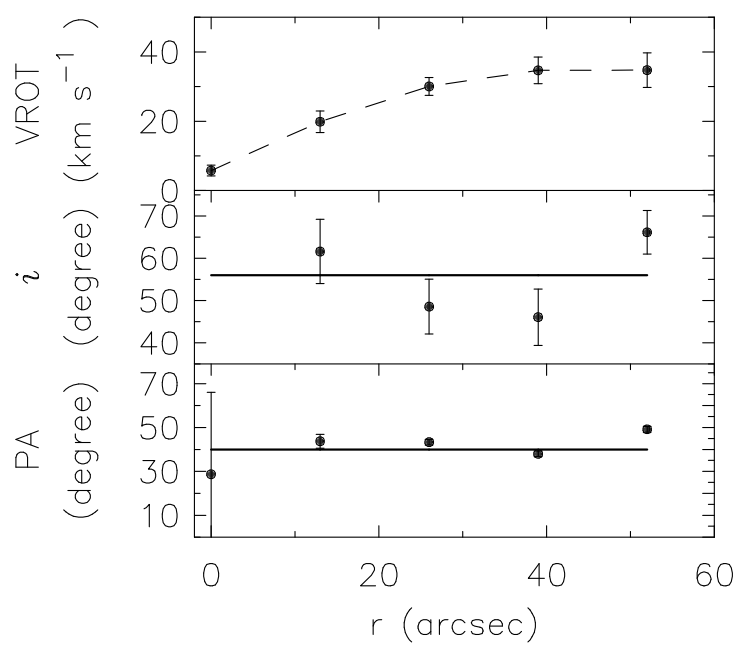

Fig. 2. Tilted-ring analysis of D512-2. The layout is identical to that of Fig. 1.

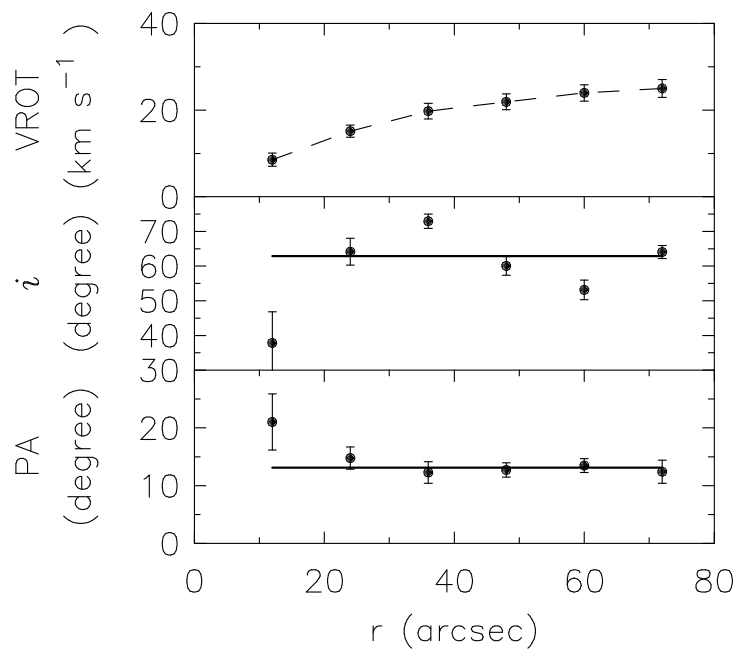

Fig. 3. Tilted-ring analysis of D564-8. The layout is identical to that of Fig. 1.

the global H I profile can be traced also in the $p V$-diagram. The maximum rotation velocity, obtained from the $p V$-diagram is $V_{p v} \sim 29 \mathrm{~km} \mathrm{~s}^{-1}$. We use ellipse fitting to the zeroth moment map to derive initial estimates for our tilted-ring fits. We set the width of the tilted-rings to $12^{\prime \prime}$. In the first tilted-ring fit, we derive a systemic velocity of $V_{\text {sys }} \approx 478 \mathrm{~km} \mathrm{~s}^{-1}$, which agrees well with the central velocity of the $W_{50}$ profile. In a subsequent fit, we derive the dynamical center by averaging the values for $x_{0}$ and $y_{0}$ over the entire radial range. The resulting center (cf. Table 3 ) agrees to within a few arcseconds with the optical center from NED. In two subsequent tilted-ring fits, we derive the PA and inclination by averaging the individual measurements over the radial range, excluding the innermost (deviant) data point. The resulting inclination $\left(i_{\text {kin }} \sim 63^{\circ} \pm 7^{\circ}\right)$ is larger than both the one derived from the $\mathrm{HI}$ disk $\left(i_{\mathrm{HI}} \sim 50^{\circ}\right)$, and the optical inclination $\left(i_{\text {opt }} \sim 35^{\circ}\right.$, Pildis et al. 1997). However, an inclination of $50^{\circ}$, or even $35^{\circ}$ can be ruled out by our kinematic data. Keeping all parameters except the rotation velocity fixed to their best estimates, we derive the rotation curve of D564-8. The maximum rotation velocity is $V_{v f} \sim 25 \mathrm{~km} \mathrm{~s}^{-1}$ (see Fig. 3). 


\section{6. $D 565-5$}

The WSRT observations of D565-5 do not detect any emission at the reported position and redshift. We do, however, see strong emission from the nearby galaxy NGC 2903 at the edge of the primary beam. The agreement of the apparent velocity of this emission with that of the Schombert et al. (1997) detection suggests that the latter detection was simply NGC 2903 emission that was picked up with the larger single-dish beam.

\subsection{D572-5 (Data presented in Fig. A.5)}

D572-5 is an irregular galaxy at a distance of $14.6 \pm 3.2 \mathrm{Mpc}$. Our H I data of this galaxy are barely resolved (see, e.g., the moment maps in Fig. A.5), which makes it difficult to derive a reliable $\mathrm{HI}$ inclination. We therefore adopt the value of the $I$-band inclination $i_{\mathrm{opt}} \sim 50^{\circ}$ as given by Pildis et al. (1997) in further analysis. Due to the low resolution, substantial off-axis H I emission enters the beam, causing the large velocity range in the minor axis $p V$-diagram. Given these problems, a rotation curve or a $V_{p v}$ from the major axis position-velocity diagram cannot be reliably derived. The only usable indicators for the maximum rotation velocity are $W_{20}$ and $W_{50}$.

\subsection{D575-1 (Data presented in Fig. A.6)}

D575-1, also known as IC 3810, is classified as an Sm/Irr galaxy. We assume a distance of $10.0 \pm 3.6 \mathrm{Mpc}$. Its global H I profile is well described by a Gaussian. The first-moment map and the position-velocity diagrams show only a small velocity range and it proved impossible to estimate either $V_{v f}$ or $V_{p v}$. The inclination of the H I disk (derived using ellipse fitting and corrected for the beam size) is $i_{\mathrm{HI}} \sim 53^{\circ}$ and in reasonable agreement with the optical inclination $\left(i_{\mathrm{opt}} \sim 61^{\circ}\right)$ from Pildis et al. (1997) (though the observed small velocity range in the velocity field and $p V$ diagram casts some doubt on the validity of these values).

\subsection{D575-2 (Data presented in Figs. 4, A.7, and A.16)}

D575-2, or UGC 8011, is a galaxy of the Hubble type Im, with an assumed distance of $12.2 \pm 4.5 \mathrm{Mpc}$. It was not possible to estimate $V_{p v}$ from the position-velocity diagram. The galaxy is kinematically lopsided, which can be seen in the differences in the velocity contours between the approaching and receding side. Using ellipse fitting, we determine the inclination of the $\mathrm{HI}$ disk to be $i_{\mathrm{HI}} \sim 52^{\circ}$, which is somewhat less inclined than what Pildis et al. (1997) found optically $\left(i_{\mathrm{opt}} \sim 63^{\circ}\right)$. With the $\mathrm{H}$ I ellipse fitting results as initial estimates for a tilted-ring analysis and adopting a width of the tilted-rings of $14^{\prime \prime}$, we determine a systemic velocity of $V_{\text {sys }} \sim 774 \mathrm{~km} \mathrm{~s}^{-1}$. This agrees to within $2 \mathrm{~km} \mathrm{~s}^{-1}$ with the center of the $W_{50}$ profile. Fixing the systemic velocity, we determine a position of the dynamical center (see Table 3) in excellent agreement with the optical center as given in NED. Leaving PA and $i$ unconstrained, we find a gradual decrease of the position angle from $\sim 230^{\circ}$ in the inner parts to $\sim 210^{\circ}$ in the outskirts of D575-2. Fixing the PA to these values, we derive the inclination by averaging over all data points. The resulting inclination $\left(i_{\text {kin }} \sim 57^{\circ} \pm 5^{\circ}\right)$ is halfway between the optical inclination and the one from the H I disk. Keeping the inclination fixed to the kinematic estimate, we derive the rotation curve of D575-2 (see Fig. 4). After a linear increase in the inner parts of the galaxy, the rotation velocity reaches a flat part at $\sim 74 \mathrm{~km} \mathrm{~s}^{-1}$.

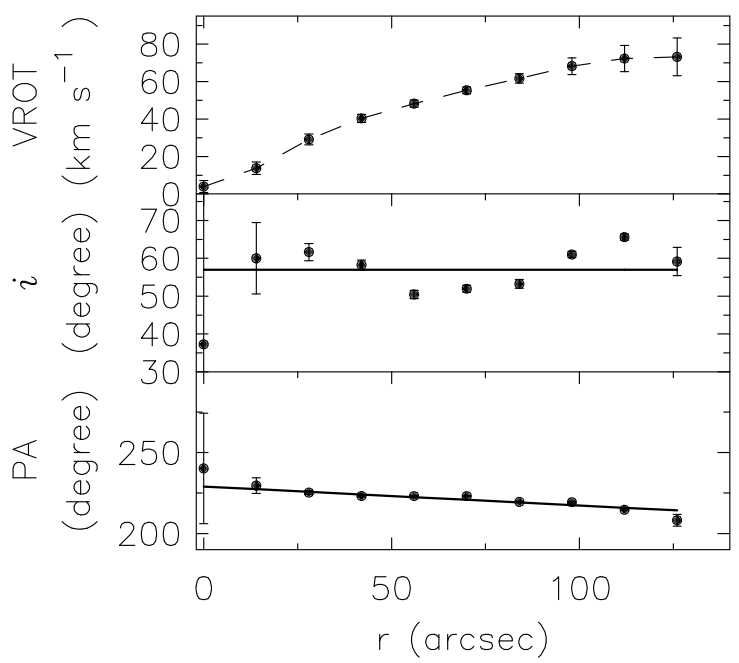

Fig. 4. Tilted-ring analysis of D575-2. The layout is identical to that of Fig. 1.

\subsection{D575-5 (Data presented in Fig. A.8)}

D575-5 is classified as a dwarf irregular at an assumed distance of $7.7 \pm 3.1 \mathrm{Mpc}$. Its global Hi profile has a Gaussian shape and its $W_{50}$ is the smallest of our sample $\left(W_{50} \sim 19 \mathrm{~km} \mathrm{~s}^{-1}\right.$ for the Hanning smoothed data cube). Pildis et al. (1997) estimate the optical inclination to be $i_{\text {opt }} \sim 66^{\circ}$, which is higher than the (beam corrected) inclination of the H I disk, for which we derive $i_{\mathrm{HI}} \sim 50^{\circ}$. However, neither the velocity field, nor the major axis $p V$-diagram show clear signs of rotation. We were unable to derive a maximum rotation velocity from either the velocity field, or from the major-axis position-velocity diagram. The secondmoment map shows values of $6-8 \mathrm{~km} \mathrm{~s}^{-1}$ throughout the entire disk. This suggests D575-5 is close to face-on and that a relatively large fraction of the profile width is caused by turbulence. For the inclination correction of $W_{20}$ and $W_{50}$, we use the inclination of the H I disk, as the appearance of D575-5 rules out the optical inclination of $i_{\text {opt }} \sim 66^{\circ}$, though we note that the observed small velocity range indicates that even this $\mathrm{H}$ I value is likely to be an overestimate of the true inclination.

\subsection{D631-7 (Data presented in Figs. 5, A.9, and A.17)}

D631-7 is also known as UGC 4115 and is classified as a dwarf irregular. Karachentsev et al. (2003) estimate its distance to be $5.5 \pm 0.6 \mathrm{Mpc}$ using the luminosity of the tip of the red giant branch. The global H I profile of D631-7 is single-peaked. Its velocity field is well-resolved and shows clear signs of rotation. The major axis $p V$-diagram shows indications of a flat rotation curve in the outer parts of the galaxy. Ellipse fitting yields an inclination of the $\mathrm{HI}$ disk $i_{\mathrm{HI}} \sim 57^{\circ}$. The ellipse fit results are used as initial estimates for the tilted ring analysis. The width of the rings is set to $12^{\prime \prime}$. In the first tilted-ring fit, we derive a systemic velocity of $V_{\text {sys }} \sim 311 \mathrm{~km} \mathrm{~s}^{-1}$, which agrees well with the center of the $W_{50}$ profile. The dynamical center is fitted in the second run with ROTCUR. The resulting center position is given in Table 3, and agrees to within 1 " with the optical center as given by NED. The PA of D631-7 is obtained in a subsequent fit with ROTCUR and shows a gradual increase from $\sim 318^{\circ}$ in the inner parts to $\sim 330^{\circ}$ in the outer parts. Fixing the PA to these values, we derive the kinematic inclination of D631-7 to be $i_{\text {kin }} \sim 59^{\circ} \pm 3^{\circ}$ by averaging the individual tilted-ring values for $50^{\prime \prime} \leq r \leq 150^{\prime \prime}$. This is in good agreement with the 


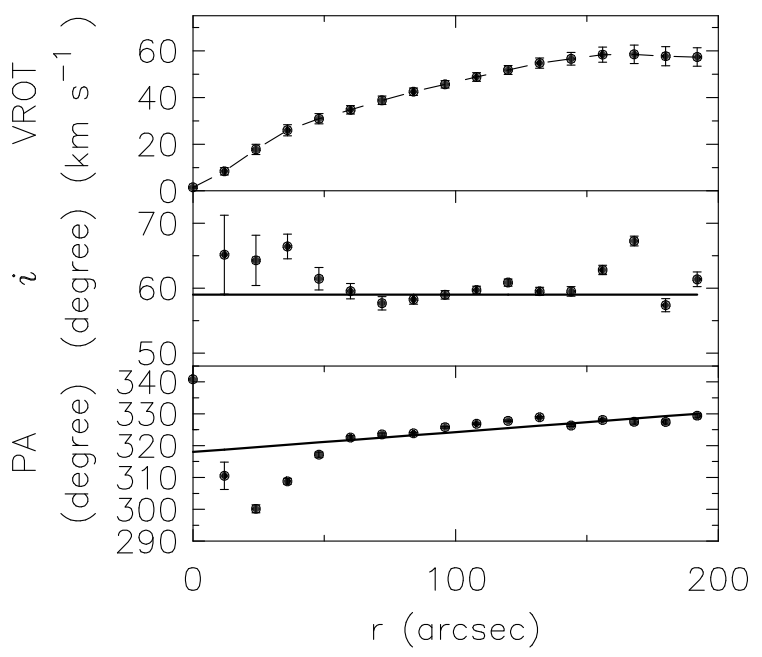

Fig. 5. Tilted-ring analysis of D631-7. The layout is identical to that of Fig. 1.

inclination of the $\mathrm{HI}$ disk $\left(i_{\mathrm{HI}} \sim 57^{\circ}\right)$, but more face-on than the optical inclination $\left(i_{\mathrm{opt}} \sim 66^{\circ}\right.$, derived by Pildis et al. 1997). Keeping all parameters except VROT fixed, we determine the rotation curve of D631-7 (see Fig. 5). It shows the typical solid-body rotation in the inner parts, but reaches a flat part at $V_{v f} \sim 58 \mathrm{~km} \mathrm{~s}^{-1}$. Assuming the kinematical inclination we derive $V_{p v} \sim 53 \mathrm{~km} \mathrm{~s}^{-1}$ from the $p V$ diagram.

\subsection{D640-13 (Data presented in Fig. A.10)}

D640-13 is an Im/Sm type galaxy with an adopted distance of $13.4 \pm 3.4 \mathrm{Mpc}$. Its global H I profile has a Gaussian shape. Comparing the size and the shape of the beam with that of the moment maps shows that the galaxy is barely resolved. This heavily affects the derived inclination of the $\mathrm{HI}$ disk $\left(i_{\mathrm{HI}} \sim 71^{\circ}\right)$ which is much higher than the optical inclination $\left(i_{\text {opt }} \sim 48^{\circ}\right)$ by Pildis et al. (1997). We therefore use the optical inclination for our further analysis. As neither the position-velocity diagrams, nor the first-moment map show clear signs of rotation, only the widths of the $W_{20}$ and $W_{50}$ profiles remain as a proxy for the maximum rotation velocity, which we correct using the optical inclination.

\subsection{D646-7 (Data presented in Fig. A.11)}

D646-7 (or UGC 8091) is classified as an irregular dwarf galaxy for which Karachentsev et al. (2003) estimate a distance of $2.1 \pm 0.6 \mathrm{Mpc}$, using the tip of the red giant branch. The global H I profile of D646-7 has a clear Gaussian shape. The galaxy lacks clear signs of rotation, both in the velocity field and in the major-axis position-velocity diagram. Thus, only $W_{20}$ and $W_{50}$ remain as indicators for the maximum rotation velocity. The optical inclination of D646-7, derived by Pildis et al. (1997), is $i_{\text {opt }} \sim 55^{\circ}$, which is in good agreement with the (uncorrected) inclination of the $\mathrm{HI}$ disk $\left(i_{\mathrm{HI}} \sim 51^{\circ}\right)$. However, given that the galaxy is not well resolved, we use the optical inclination in our analysis.

\section{Analysis}

In the previous section, we have derived several estimates for the maximum rotation velocities of the galaxies in our sample.

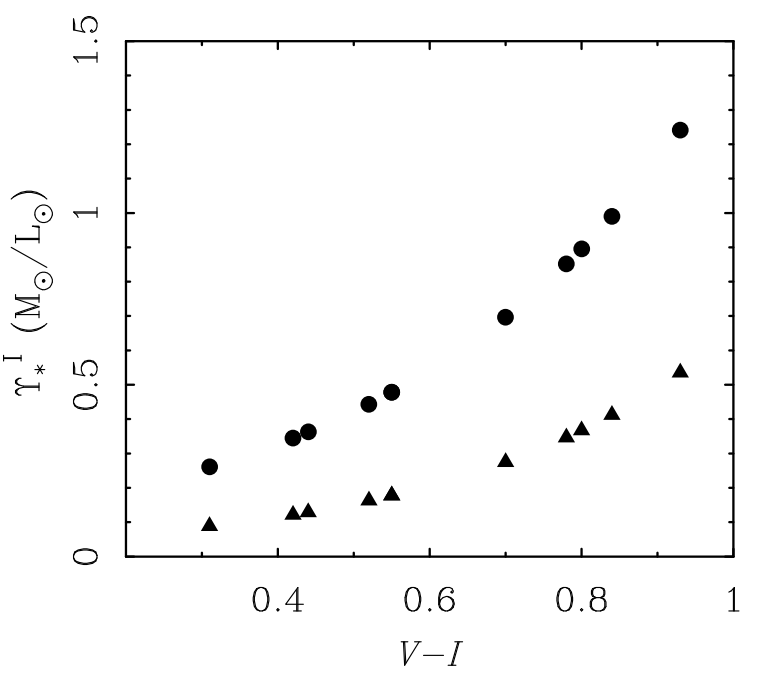

Fig. 6. Stellar $I$-band mass-to-light ratio $\left(\Upsilon_{*}\right)$ vs. $(V-I)$ color for two different population synthesis models. The circles are based on the models from Bell \& de Jong (2001) with a scaled Salpeter IMF, the triangles are based on the Portinari et al. (2004) models with a Kroupa IMF. Note that D631-7 and D640-13 have the same color: for clarity reasons we only plot one point.

Before using them to construct a BTF, we discuss our choices for the stellar mass-to-light ratios $\left(\Upsilon_{*}\right)$, describe the corrections applied to the line width measurements and estimate the uncertainties in the rotation velocities and baryonic masses.

\subsection{The choice of the stellar mass-to-light ratio $\Upsilon_{*}$}

One of the largest contributors to the scatter in baryonic mass is the assumed value for the stellar mass-to-light ratio $\Upsilon_{*}$ and its uncertainty. Fortunately, the effect of $\Upsilon_{*}$ becomes less important when dealing with low-mass galaxies, as their stellar mass generally contributes less to the total baryonic mass than the gas mass does (for reasonable values of $\Upsilon_{*}$ ). The $\Upsilon_{*}$ values adopted here are derived on the basis of two different population synthesis models using two different Initial Mass Functions (IMFs). The first model is from Bell \& de Jong (2001) and uses a scaled Salpeter IMF (Salpeter 1955). The second population synthesis model discussed here uses a Kroupa IMF (Kroupa 1998) and is taken from Portinari et al. (2004). Figure 6 shows the derived stellar (I-band) mass-to-light ratios of our galaxies for the two population models as a function of color.

It is immediately apparent that the $\Upsilon_{*}$ from Bell \& de Jong (2001) are consistently larger than the ones from Portinari et al. (2004). Although this difference can amount to a factor close to three, the impact on the baryonic mass (i.e., the sum of the stellar mass and the gas mass) is rather small, as can be seen in Fig. 7 , where, for each galaxy, we compare the ratio of the two different stellar mass assumptions against the baryonic mass implied by the respective $\Upsilon_{*}$ values. In the following analysis we will adopt the average $\Upsilon_{*}$ values implied by the two models. The individual mass-to-light ratios are listed in Table 4.

\subsection{Line width corrections}

The rotation velocities derived using the profile widths $W_{20}$ and $W_{50}$, still need to be corrected for instrumental velocity 
Table 4. Stellar, gas, and baryonic masses.

\begin{tabular}{|c|c|c|c|c|c|c|c|}
\hline ID & $\begin{array}{c}\Upsilon_{*}^{I}(\text { Bell }) \\
(2)\end{array}$ & $\Upsilon_{*}^{I}$ (Portinari) & $\left\langle\Upsilon_{*}^{I}\right\rangle$ & $\begin{array}{c}M_{\mathrm{gas}} \\
10^{8} M_{\odot} \\
(5)\end{array}$ & $\begin{array}{c}M_{*}(\text { Bell }) \\
10^{8} M_{\odot} \\
\quad(6)\end{array}$ & $\begin{array}{c}M_{*} \text { (Portinari) } \\
10^{8} M_{\odot} \\
(7)\end{array}$ & $\begin{array}{c}\left\langle M_{\mathrm{bar}}\right\rangle \\
10^{8} M_{\odot} \\
\quad(8)\end{array}$ \\
\hline D500-2 & 0.35 & 0.12 & $0.24 \pm 0.12$ & 12.19 & 0.73 & 0.26 & 12.69 \\
\hline D500-3 & 0.26 & 0.09 & $0.18 \pm 0.09$ & 0.92 & 0.28 & 0.09 & 1.11 \\
\hline D512-2 & 0.90 & 0.37 & $0.64 \pm 0.27$ & 0.98 & 0.93 & 0.38 & 1.63 \\
\hline D564-8 & 1.24 & 0.54 & $0.89 \pm 0.35$ & 0.22 & 0.14 & 0.06 & 0.32 \\
\hline D572-5 & 0.44 & 0.16 & $0.30 \pm 0.14$ & 1.20 & 0.19 & 0.07 & 1.33 \\
\hline D575-1 & 0.70 & 0.28 & $0.49 \pm 0.21$ & 0.53 & 0.26 & 0.10 & 0.71 \\
\hline D575-2 & 1.07 & 0.45 & $0.76 \pm 0.31$ & 4.41 & 1.02 & 0.43 & 5.13 \\
\hline D575-5 & 0.36 & 0.13 & $0.25 \pm 0.12$ & 0.60 & 0.04 & 0.02 & 0.63 \\
\hline D631-7 & 0.48 & 0.18 & $0.33 \pm 0.15$ & 2.05 & 0.20 & 0.08 & 2.19 \\
\hline D640-13 & 0.48 & 0.18 & $0.33 \pm 0.15$ & 0.64 & 0.18 & 0.07 & 0.76 \\
\hline D646-7 & 0.99 & 0.41 & $0.70 \pm 0.29$ & 0.05 & 0.09 & 0.04 & 0.12 \\
\hline
\end{tabular}

Notes: (1) galaxy identifier; (2) stellar I-band mass-to-light ratio based on Bell \& de Jong (2001); (3) stellar I-band mass-to-light ratio based on Portinari et al. (2004); (4) average of $I$-band $\Upsilon_{*}$ values listed in Cols. (2) and (3). For the uncertainty in $\Upsilon_{*}$, we assume half the difference between the mass-to-light ratios of the two population models; (5) the gas mass in $10^{8} M_{\odot}$; (6) the stellar mass based on $\Upsilon_{*}$ in Col. (2) in units of $10^{8} M_{\odot}$; (7) like (6), but based on $\Upsilon_{*}$ in Col. (3); (8) the baryonic mass used in further analysis, derived from the gas mass in Col. (5) and the average of the stellar masses in Cols. (6) and (7).

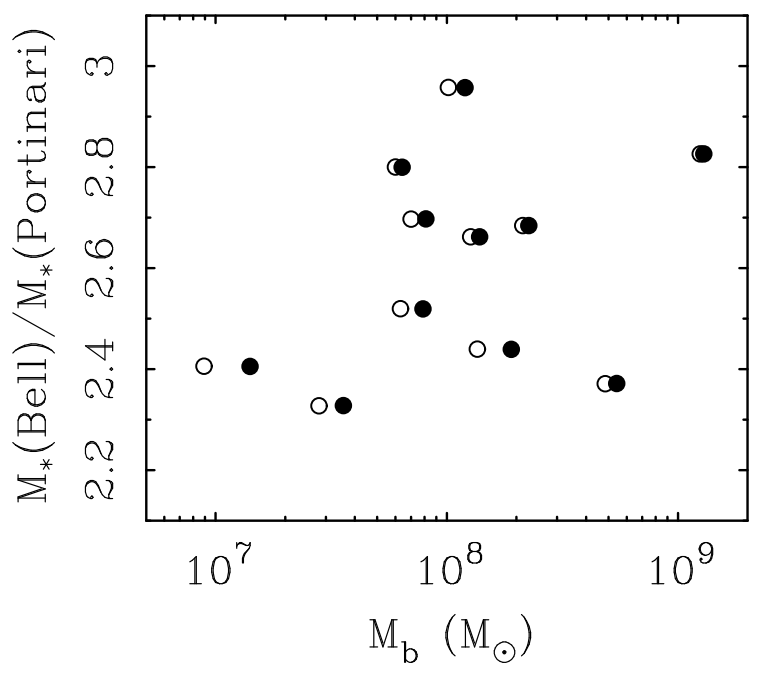

Fig. 7. The ratio of the stellar mass from Bell \& de Jong (2001) and Portinari et al. (2004) vs. the implied baryonic mass. Baryonic masses derived using the Bell \& de Jong (2001) stellar masses are shown as filled circled; baryonic masses derived using the Portinari et al. (2004) models as open circles. It is clear the choice of $\Upsilon_{*}$ has only a small effect on the baryonic masses.

resolution and turbulence. To correct for the instrumental resolution, we use the approach of Verheijen (1997):

$W_{x}=W_{x}^{\mathrm{obs}}-C_{x} \cdot\left[\sqrt{1+\left(\frac{R}{23.5}\right)^{2}}-1\right]$,

where the subscript $x$ refers to the chosen profile width measure (i.e., $x=20$ for $W_{20}$ and $x=50$ for $\left.W_{50}\right), R$ is the instrumental resolution in $\mathrm{km} \mathrm{s}^{-1}$ (cf. Col. (9) in Table 1), and $C_{x}$ is a constant, equal to $C_{20}=35.8$ for the $W_{20}$ profile and $C_{50}=23.5$ for the $W_{50}$ profile. The "obs" superscript in $W_{x}^{\text {obs }}$ denotes the observed profile width (given in Cols. (9) and (10) of Tables 2 and 3).

In addition to broadening due to finite velocity resolution, we correct the velocity widths for broadening due to turbulent motion of the neutral gas. Following Tully \& Fouque (1985), we use

$W_{x, \text { turb }}^{2}=W_{x}^{2}+W_{t, x}^{2}\left[1-2 e^{-\left(\frac{W_{x}}{W_{c, x}}\right)^{2}}\right]-2 W_{x} W_{t, x}\left[1-\mathrm{e}^{-\left(\frac{W_{x}}{W_{c, x}}\right)^{2}}\right]$

The subscript $x$ again refers to the profile width measured at 20 or 50 percent of the peak flux; $W_{x}$ indicates velocity widths already corrected for instrumental resolution following Eq. (5). The $W_{c, x}$ values represent the typical velocity widths where the shapes of the velocity profiles change from boxy (doublehorned) to Gaussian; the constants $W_{t, x}$ indicate the amount by which turbulent motion of the neutral gas broadens the H I profile. For the choice of $W_{c, x}$ and $W_{t, x}$, we follow Verheijen (1997), who assumes a turbulent motion of the gas with a velocity dispersion of $10 \mathrm{~km} \mathrm{~s}^{-1}$ and values of $W_{t, 20}=22 \mathrm{~km} \mathrm{~s}^{-1}$, $W_{t, 50}=5 \mathrm{~km} \mathrm{~s}^{-1}, W_{c, 20}=120 \mathrm{~km} \mathrm{~s}^{-1}$ and $W_{c, 50}=100 \mathrm{~km} \mathrm{~s}^{-1}$ (see Chap. 5 of Verheijen 1997 for an extensive discussion of these correction terms). The resulting corrected profile widths are given in Cols. (11) and (12) of Tables 2 and 3. Note that the profile widths listed in Tables 2 and 3 are not corrected for inclination.

\subsection{Sources of uncertainties}

\subsubsection{Uncertainty in $V_{\max }$}

The uncertainty in $V_{\max }$ depends on the measurement error in the observed velocity and that in the inclination value. For the velocity uncertainty we assume that we can measure the velocity with an accuracy of one channel width $\left(\sim 4 \mathrm{~km} \mathrm{~s}^{-1}\right)$. In addition, we assume that noise in the data or intrinsic variance within the galaxies introduce another channel width worth of uncertainty. The total uncertainty we assume is the quadratic sum of these two terms or $6 \mathrm{~km} \mathrm{~s}^{-1}$.

For the uncertainty in the derived inclination angle, we distinguish between galaxies with a kinematically derived inclination, and those where we had to use the optical inclination or that of the H I disk. For the former category, we adopt the scatter of the inclination values of the individual tilted-ring models (see middle panels in Figs. 1-5) as the inclination uncertainty (see Col. (13) of Table 3). The average value of the uncertainties in these kinematically derived inclinations is $6^{\circ}$ and is used as 
the uncertainty in the inclination angles of the remaining galaxies. The total uncertainty of the (inclination-corrected) maximum rotation velocity is then calculated using Gaussian error propagation.

\subsubsection{Uncertainty of the baryonic mass}

The baryonic mass is the sum of the stellar mass and the gas mass:

$M_{\mathrm{bar}}=M_{\mathrm{stars}}+M_{\mathrm{gas}}$.

The stellar mass is calculated through

$M_{\text {stars }}=\Upsilon_{*}^{I} 10^{-0.4\left[M_{V}-(V-I)-4.02\right]}$,

where $\Upsilon_{*}^{I}$ is the stellar mass-to-light ratio in the $I$-band, $M_{I}=$ $M_{V}-(V-I)$ is the absolute $I$-band magnitude, and 4.02 is the magnitude of the Sun in the I-band. The absolute magnitude of a galaxy depends through the distance modulus on its apparent magnitude and its distance. Thus, the stellar mass depends on three quantities: stellar mass-to-light ratio, distance, and apparent magnitude. For the uncertainty in the mass-to-light ratio, we use the values derived in Sect. 5.1. For the uncertainty in the distances, we use the values listed in Sect. 4. The apparent magnitudes of the galaxies in our sample were determined by Pildis et al. (1997), who report a photometric accuracy of 0.05 mag. This is insignificant compared to the influence of the uncertainties in the distance and in the stellar $\Upsilon_{*}$, and we therefore ignore the uncertainties of $m_{V}$ for the uncertainty of the stellar mass.

The second term contributing to the baryonic disk mass is $M_{\mathrm{gas}}$, the mass of the gas, which is given by:

$M_{\mathrm{gas}}=1.4 M_{\mathrm{HI}}=1.4 \cdot 2.36 \cdot 10^{5} D^{2} \int S \mathrm{~d} v$,

where $M_{\mathrm{HI}}$ is the total H I mass, $D$ is the distance in Mpc, $S$ is the total flux in mJy beam ${ }^{-1}$, and $\mathrm{d} v$ is the velocity resolution in $\mathrm{km} \mathrm{s}^{-1}$. The constant factor 1.4 corrects the $\mathrm{H}$ I mass for the presence of helium and metals. Note that since $\mathrm{H}_{2} / \mathrm{HI}$, the ratio between the molecular and the neutral hydrogen, is much lower in dwarf galaxies compared to luminous spirals (Taylor et al. 1998; Leroy et al. 2005), we do not apply correction terms to account for molecular hydrogen. The uncertainty of the H I mass depends on the uncertainty of the total flux, and quadratically on the distance uncertainty. We focus again on the uncertainty in the distance, which is the dominant source of uncertainty here. Inserting Eqs. (8) and (9) into Eq. (7), we determine the uncertainty of the baryonic mass by assuming a Gaussian error propagation of the individual uncertainties discussed above. The stellar, gas, and baryonic masses are also listed in Table 4.

\subsection{The baryonic Tully-Fisher relation}

In this section we present the baryonic Tully-Fisher relation for the galaxies of our sample, using the different estimates for $V_{\max }$ derived previously. As a reference, we use the work by McGaugh (2005). It presents the BTF for galaxies with well-determined rotation velocities between $50 \mathrm{~km} \mathrm{~s}^{-1}$ and $300 \mathrm{~km} \mathrm{~s}^{-1}$, and tests several methods to determine stellar mass-to-light ratios. The one yielding the smallest scatter in the BTF is based on the massdiscrepancy-acceleration relation (MDAcc, see McGaugh 2004) and gives a BTF relation

$M_{\mathrm{bar}}=50 V_{\max }^{4}$, with the baryonic mass in $M_{\odot}$ and the maximum rotation velocity in $\mathrm{km} \mathrm{s}^{-1}$.

For the construction of a BTF relation using our galaxies, we use the four different estimates for $V_{\max }$ derived earlier. To recapitulate, these are:

(a) $V_{v f}$, obtained from a tilted-ring analysis of the velocity fields;

(b) $V_{p v}$, obtained from fitting the outer parts in major-axis position-velocity diagrams;

(c) $V_{W 20}$, obtained from $\frac{1}{2} W_{20}$, the rotation velocity as derived from half the (corrected) width of the H I profile at the 20 percent level of the maximum flux;

(d) $V_{W 50}$, obtained from $\frac{1}{2} W_{50}$, the rotation velocity as derived from half the (corrected) width of the H I profile at the 50 percent level of the maximum flux.

These velocities are all corrected for inclination. Additional corrections have been applied to $V_{W 20}$ and $V_{W 50}$ (as described in Sect. 5.2).

In Fig. 8, we show the baryonic Tully-Fisher relation for the galaxies of our sample using the different velocity estimates. The uncertainties are discussed in Sect. 5.3. In Fig. 8, we distinguish between galaxies in which clear signatures of rotation were detected in the position-velocity diagram and/or the velocity field ( $V_{p v}$ and/or $V_{v f}$ available in addition to $V_{W 20}$ and $V_{W 50}$; the rotation curve sub-sample), and galaxies for which no clear rotation was detectable (only $V_{W 20}$ and $V_{W 50}$ available; the profile-width sub-sample). For all four measures of $V_{\max }$, the galaxies in the rotation curve sub-sample are consistent with the BTF as derived in McGaugh (2005) and Stark et al. (2009).

A few galaxies in the profile-width sub-sample are also consistent with a line-width-based BTF, but the majority of these galaxies are found to the left of the extrapolated BTF. This may indicate an increased scatter at the low line-width end of a line-width based BTF. Alternatively, the galaxies may be more face-on than indicated by the optical or H I inclinations (e.g., D575-5), or they may not be supported by rotation. Without independent and/or resolved measures of the rotation velocity and the kinematic inclinations, it is difficult to say anything further on these galaxies.

In the following we therefore restrict our analysis to those galaxies which have a well resolved velocity field which allowed us to derive a rotation curve or - at the very least - a maximum rotation velocity using the position-velocity diagram (consistent with the procedure used in McGaugh 2005 and Stark et al. 2009). We emphasize that this is our only selection criterion. We do not preferentially select against galaxies that are not on the BTF, (in fact, one of the profile-width-only galaxies (D572-5) is right on the BTF), and we include all galaxies for which $V_{p v}$ or $V_{v f}$ could be determined regardless of their position in the BTF diagram.

\subsection{The scatter of the BTF}

The panels in Fig. 8 clearly show that the data points from our rotation curve sample agree well with the BTF relation from McGaugh (2005) as given in Eq. (10). This relation was derived using resolved observations of the flat parts of the rotation curves of the sample galaxies. Including our five galaxies with measured values of $V_{v f}$ yields a revised BTF of the form

$M_{\mathrm{bar}}=58 V_{\max }^{3.97}$. 

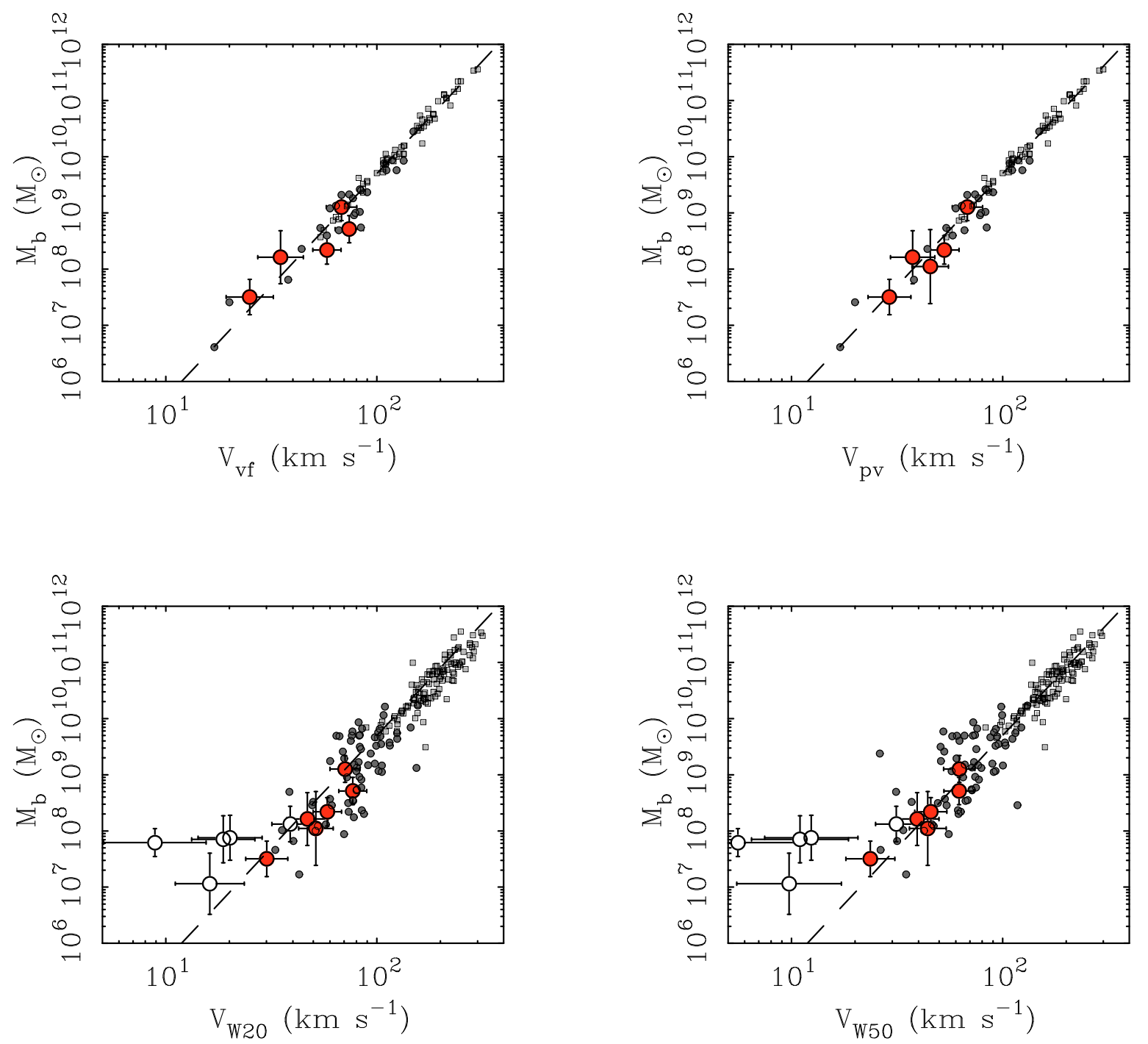

Fig. 8. The baryonic Tully-Fisher relation using four different estimates for $V_{\max }$, namely $V_{v f}$ from the tilted-ring analysis of the velocity fields (top left), $V_{p v}$ from the major-axis position-velocity diagram (top right), $V_{W 20}$, i.e., half the $W_{20}$ (bottom left), and $V_{W 50}$, i.e., half the $W_{50}($ bottom right). As a reference, we show the BTF relation of the analysis of McGaugh (2005, dashed line). In the top panels, the star-dominated galaxies of McGaugh (2005) are shown as small squares and the gas-dominated galaxies of Stark et al. (2009) are shown as small circles. In the lower panels, the velocity widths from Bothun (1985) (small squares) and McGaugh et al. (2000) (small circles) are shown for comparison. Our new data are show as large circles. Galaxies for which no rotation is discernable are shown as open circles (with line-widths only) and those with rotation detected in the position-velocity diagram are shown as filled circles. The latter fall along the extrapolation of the BTF relation found by McGaugh (2005) while some of the former fall off the relation. These galaxies may not be supported by rotation, may have incorrect inclinations, or could simply reflect the increased scatter in the relation when line-width is used as a proxy for rotation velocity.

This is remarkably close to the revised BTF presented in Stark et al. (2009), which extends the McGaugh (2005) BTF with an independent sample of low-mass gas-rich galaxies. They find

$M_{\text {bar }}=62 V_{\max }^{3.94}$.

The three fits given in Eqs. (10), (11) and (12) are statistically identical.

For comparison, Bell \& de Jong (2001) find a slope of 3.5 for a subsample of the McGaugh (2005) sample, but choose different $\Upsilon_{*}$ values than McGaugh (2005) does. Using identical $\Upsilon_{*}$ values yields identical slopes. Work by Geha et al. (2006) gives a slope of 3.7, De Rijcke et al. (2007) find a slope of 3.15, but this includes early-type galaxies. An analysis by Meyer et al. (2008) of H I-selected galaxies gives a steeper slope of 4.35. The majority of these studies deal with massive galaxies, dominated by stars, and are therefore sensitive to assumptions on $\Upsilon_{*}$. The new data presented here provide a test of the slope extrapolated from the fit to higher mass galaxies that do not overlap with this sample, and prefer a slope of $\sim 4$. This is also the conclusion of Stark et al. (2009) who calibrate the BTF using only gas-dominated galaxies, independent of assumptions on $\Upsilon_{*}$. They find a slope of 3.94 (Eq. (12)). If a single, unbroken BTF exists, this can only imply that the slope at the high mass end must also be close to 4 .

We now return to the conclusions of Franx \& de Zeeuw (1992), who use the scatter of the TF relation to put constraints on the ellipticity of dark matter halos. They argue that if the potential in the plane of the disk is elongated, then the different viewing angles will cause scatter in the TF relation. In return, the scatter of the (B)TF relation can be used to put an upper limit on the ellipticity of the potential in the plane of the disk. According to Franx \& de Zeeuw (1992), a TF relation with a scatter of $0.31 \mathrm{mag}(0.46 \mathrm{mag}$ when photometric inclinations are used) constrains the ellipticities of the potentials to be 
below 0.10 . As it is unlikely that all the scatter in the TF relation is due to different viewing angles, they argue that an elongation between 0 and 0.06 is reasonable.

Since the work of Franx \& de Zeeuw (1992), the quantity and quality of the data have improved significantly and we can now trace the BTF relation over a large range of galaxy masses and rotation velocities. The scatter in the optimum BTF from McGaugh (2005) is $\sigma=0.098 \mathrm{dex}$, or $0.25 \mathrm{mag}$. Including our galaxies results in a slightly larger scatter $\left(\sigma=0.13\right.$ or $\sigma_{\mathrm{M}}=$ $0.33 \mathrm{mag}$ ) - mainly because the distances to our galaxies are more uncertain. This scatter is only marginally larger than the scatter on which Franx \& de Zeeuw (1992) based their upper limits for the ellipticity of the potentials in the disk plane.

Their arguments equally apply to our sample. As the small scatter in the BTF holds down to rotation velocities of $\sim 25 \mathrm{~km} \mathrm{~s}^{-1}$, it follows that the elongation of the potentials of these galaxies cannot be very large, and similar limits as those derived by Franx \& de Zeeuw (1992), also apply to our galaxies. The upper limit on elongation of the potential of 0.06 is consistent with the results derived by Trachternach et al. (2008) who use harmonic decompositions of the velocity fields of 18 nearby well-resolved galaxies from the THINGS survey (Walter et al. 2008; de Blok et al. 2008) to put constraints on the elongation of the potential in the plane of the disk. They find that the the average elongation of the potential is small $(0.017 \pm 0.020)$, particularly when compared to what is found in CDM simulations (cf. Frenk et al. 1988; Hayashi \& Navarro 2006). Trachternach et al. (2008) also find that the elongation does not increase towards the center of the galaxies. The tightly correlated BTF relation presented in this paper constrains the ellipticity of galaxy potentials using a different method, but reaches similar results.

\section{Conclusions}

We present new HI observations of a sample of low-mass dwarf galaxies and use these to explore and extend the baryonic Tully-Fisher (BTF) relation at low rotation velocities and galaxy masses. We present and discuss several estimates for $V_{\max }$, the maximum rotation velocity. For galaxies where a clear rotation signal could be detected, the different estimates are in good agreement and the BTF relations based on them are equally wellconstrained. We discuss the choice of stellar mass-to-light ratio $\left(\Upsilon_{*}\right)$ and show that its choice is not crucial for the extreme dwarf galaxies in our sample, since for them, the stellar mass generally contributes less to the total baryonic mass than is the case for luminous high-mass galaxies.

The small scatter in the BTF presented here $\left(\sigma_{\mathrm{M}}=\right.$ $0.33 \mathrm{mag}$ ) puts strong constraints on the ellipticity of the potential in the plane of the disk of the galaxies. Our results are in agreement with those from Franx \& de Zeeuw (1992), Franx et al. (1994), and Trachternach et al. (2008), indicating that, at least in the plane of the disk, galaxy halos are not very elongated. The small scatter in the BTF over almost 5 orders of magnitude of baryonic mass indicates it is a fundamental relation which tightly couples the visible baryonic matter and the dark matter content of galaxies.

Acknowledgements. C.T. would like to thank Janine van Eymeren and Volker Knierim for many stimulating discussions. We also thank the anonymous referee for valuable comments. The work of C.T. is supported by the German Ministry for Education and Science (BMBF) through grant 05 AV5PDA/3. The work of W.J.G.d.B. is based upon research supported by the South African Research Chairs Initiative of the Department of Science and Technology and National Research Foundation. The Westerbork Synthesis Radio Telescope is operated by the ASTRON (Netherlands Foundation for Research in Astronomy) with support from the Netherlands Foundation for Scientific Research (NWO). This research has made use of the NASA/IPAC Extragalactic Database (NED) which is operated by the Jet Propulsion Laboratory, California Institute of Technology, under contract with the National Aeronautics and Space Administration.

\section{References}

Bell, E. F., \& de Jong, R. S. 2001, ApJ, 550, 212

Blanton, M. R., Geha, M., \& West, A. A. 2008, ApJ, 682, 861

Bothun, G. D. 1985, AJ, 90, 1982

Briggs, D. S. 1995, in BAAS, 27, 1444

Broeils, A. H. 1992, Ph.D. Thesis, Univ. Groningen

de Blok, W. J. G., McGaugh, S. S., \& van der Hulst, J. M. 1996, MNRAS, 283, 18

de Blok, W. J. G., Walter, F., Brinks, E., et al. 2008, AJ, 136, 2648

De Rijcke, S., Zeilinger, W. W., Hau, G. K. T., Prugniel, P., \& Dejonghe, H. 2007, ApJ, 659, 1172

Eder, J. A., \& Schombert, J. M. 2000, ApJS, 131, 47

Eisenstein, D. J., \& Loeb, A. 1996, ApJ, 459, 432

Franx, M., \& de Zeeuw, T. 1992, ApJ, 392, L47

Franx, M., van Gorkom, J. H., \& de Zeeuw, T. 1994, ApJ, 436, 642

Frenk, C. S., White, S. D. M., Davis, M., \& Efstathiou, G. 1988, ApJ, 327, 507

Geha, M., Blanton, M. R., Masjedi, M., \& West, A. A. 2006, ApJ, 653, 240

Gurovich, S., McGaugh, S. S., Freeman, K. C., et al. 2004, Publ. Astron. Soc. Aust., 21, 412

Hayashi, E., \& Navarro, J. F. 2006, MNRAS, 373, 1117

Karachentsev, I. D., Makarov, D. I., Sharina, M. E., et al. 2003, A\&A, 398, 479

Kovac, K. 2007, Ph.D. Thesis, Univ. Groningen

Kroupa, P. 1998, in Brown Dwarfs and Extrasolar Planets, ed. R. Rebolo, E. L.

Martin, \& M. R. Zapatero Osorio, ASP Conf. Ser., 134, 483

Leroy, A., Bolatto, A. D., Simon, J. D., \& Blitz, L. 2005, ApJ, 625, 763

Matthews, L. D., van Driel, W., \& Gallagher, III, J. S. 1998, AJ, 116, 2196

McGaugh, S. S. 2004, ApJ, 609, 652

McGaugh, S. S. 2005, ApJ, 632, 859

McGaugh, S., \& de Blok, E. 1998a, in Galactic Halos, ed. D. Zaritsky, ASP Conf. Ser., 136, 210

McGaugh, S. S., \& de Blok, W. J. G. 1998b, ApJ, 499, 41

McGaugh, S. S., Schombert, J. M., Bothun, G. D., \& de Blok, W. J. G. 1999, in BAAS, 31, 1559

McGaugh, S. S., Schombert, J. M., Bothun, G. D., \& de Blok, W. J. G. 2000, ApJ, 533, L99

Meyer, M. J., Zwaan, M. A., Webster, R. L., Schneider, S., \& Staveley-Smith, L. 2008, MNRAS, 391, 1712

Mo, H. J., Mao, S., \& White, S. D. M. 1998, MNRAS, 295, 319

Mould, J. R., Huchra, J. P., Freedman, W. L., et al. 2000, ApJ, 529, 786

Noordermeer, E., \& Verheijen, M. A. W. 2007, MNRAS, 381, 1463

Pierce, M. J., \& Tully, R. B. 1988, ApJ, 330, 579

Pildis, R. A., Schombert, J. M., \& Eder, J. A. 1997, ApJ, 481, 157

Portinari, L., Sommer-Larsen, J., \& Tantalo, R. 2004, MNRAS, 347, 691

Sakai, S., Mould, J. R., Hughes, S. M. G., et al. 2000, ApJ, 529, 698

Salpeter, E. E. 1955, ApJ, 121, 161

Sault, R. J., Teuben, P. J., \& Wright, M. C. H. 1995, in Astronomical Data Analysis Software and Systems IV, ed. R. A. Shaw, H. E. Payne, \& J. J. E. Hayes, ASP Conf. Ser., 77, 433

Schombert, J. M., Pildis, R. A., \& Eder, J. A. 1997, ApJS, 111, 233

Springob, C. M., Masters, K. L., Haynes, M. P., Giovanelli, R., \& Marinoni, C. 2007, ApJS, 172, 599

Stark, D. V., McGaugh, S. S., \& Swaters, R. A. 2009, AJ, 138, 392

Steinmetz, M., \& Navarro, J. F. 1999, ApJ, 513, 555

Taylor, C. L., Kobulnicky, H. A., \& Skillman, E. D. 1998, AJ, 116, 2746

Trachternach, C., de Blok, W. J. G., Walter, F., Brinks, E., \& Kennicutt, R. C. 2008, AJ, 136, 2720

Tully, R. B., \& Fisher, J. R. 1977, A\&A, 54, 661

Tully, R. B., \& Fouque, P. 1985, ApJS, 58, 67

Tully, R. B., \& Pierce, M. J. 2000, ApJ, 533, 744

van der Hulst, J. M., Terlouw, J. P., Begeman, K. G., Zwitser, W., \& Roelfsema, P. R. 1992, in Astronomical Data Analysis Software and Systems I, ed. D. M. Worrall, C. Biemesderfer, \& J. Barnes, ASP Conf. Ser., 25, 131

Verheijen, M. A. W. 1997, Ph.D. Thesis, Univ. Groningen

Verheijen, M. A. W. 2001, ApJ, 563, 694

Walter, F., Brinks, E., de Blok, W. J. G., et al. 2008, AJ, 136, 2563

Willick, J. A., Courteau, S., Faber, S. M., et al. 1997, ApJS, 109, 333

Zwaan, M. A., van der Hulst, J. M., de Blok, W. J. G., \& McGaugh, S. S. 1995,

MNRAS, 273, L35 


\section{Appendix A: Atlas}

The Appendix contains summary panels for all galaxies of our sample (Figs. A.1-A.11) and channel maps for the galaxies from the rotation curve sub-sample (Figs. A.12-A.17).

The summary panels consist of two rows with three panels each, and contain the following maps:

Top row: Left panel: zeroth moment map with grayscales. Unless mentioned otherwise, grayscales run from a column density of $n_{\mathrm{HI}}=1 \times 10^{19} \mathrm{~cm}^{-2}$ (white) to $n_{\mathrm{HI}}=2 \times 10^{21} \mathrm{~cm}^{-2}$ (black). The $3 \sigma$ level is indicated by the black contour. Middle panel: first moment map. The systemic velocity is indicated by the thick contour, the contours are spaced by $10 \mathrm{~km} \mathrm{~s}^{-1}$. The approaching side can be identified by the light grayscales and black contours, and the receding side by dark grayscales and white contours. Right panel: second moment map. Grayscales run from 2 to $40 \mathrm{~km} \mathrm{~s}^{-1}$. Unless mentioned otherwise, the contours levels are given at 5,10 , and $15 \mathrm{~km} \mathrm{~s}^{-1}$. For all moment maps, the beam size is indicated in the bottom right corner. Additionally, if a kinematic center has been independently derived, it is indicated in all moment maps by a cross.

Bottom row: Left panel: major axis position-velocity diagram. The position angle of the slice indicated in the top-left corner of the panel. Grayscales run from $2 \sigma$ to $30 \sigma$, and contour levels are given at $2 \sigma+n \times 4 \sigma$ (i.e., $2,6,10,14,18 \ldots$ $\sigma)$. The dashed line indicates the systemic velocity. If a $V_{p v}$ has been derived, the resulting velocities at either side of the rotation curve are indicated by arrows. Middle panel: minor axis position-velocity diagram. Grayscales and contours are identical to the major axis position-velocity diagram. Right panel: global H I profile of the Hanning smoothed data cube. 
C. Trachternach et al.: Baryonic Tully-Fisher relation, Online Material p 2

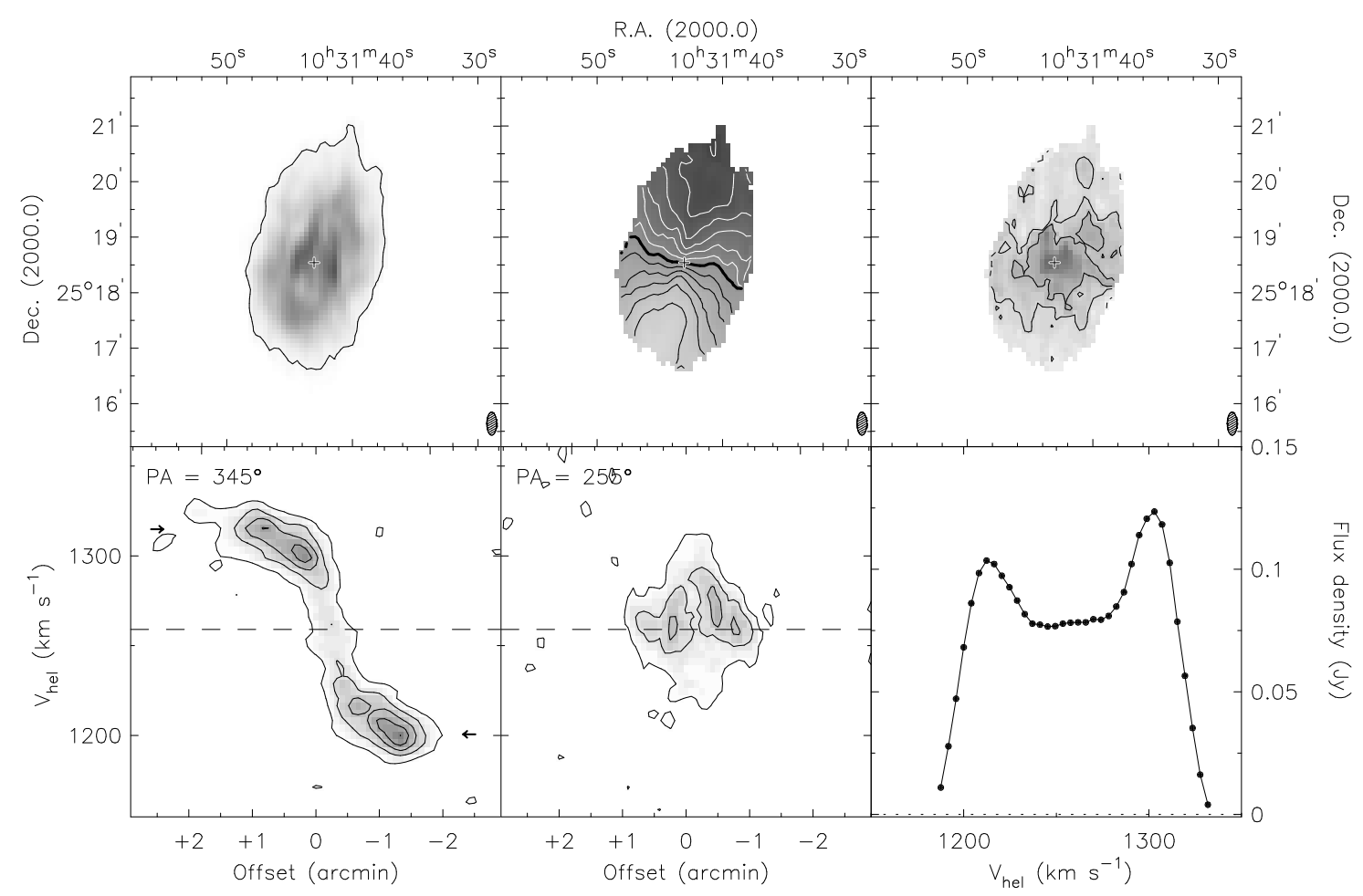

Fig. A.1. Summary panel for D500-2. A general description of the panels and levels is given at the beginning of the Appendix. The $3 \sigma$ contour in the zeroth moment map corresponds to a flux of $5.09 \mathrm{mJy}$, which translates to a column density limit of $n_{\mathrm{HI}}=8.8 \times 10^{19} \mathrm{~cm}^{-2}$.

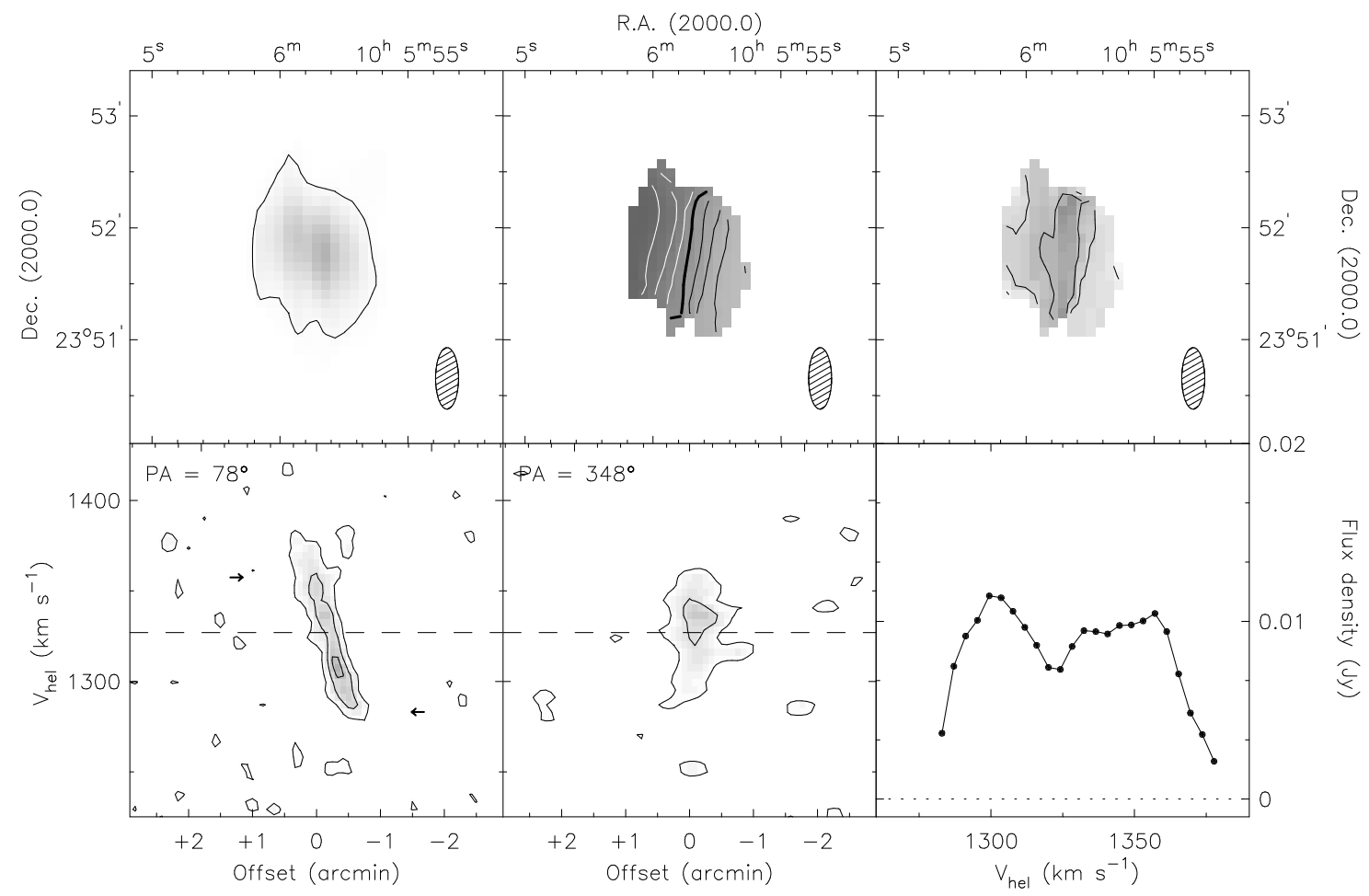

Fig. A.2. Summary panel for D500-3. A general description of the panels and levels is given at the beginning of the Appendix. The $3 \sigma$ contour in the zeroth moment map corresponds to a flux of $4.51 \mathrm{mJy}$, which translates to a column density limit of $n_{\mathrm{HI}}=5.2 \times 10^{19} \mathrm{~cm}^{-2}$. 
C. Trachternach et al.: Baryonic Tully-Fisher relation, Online Material p 3

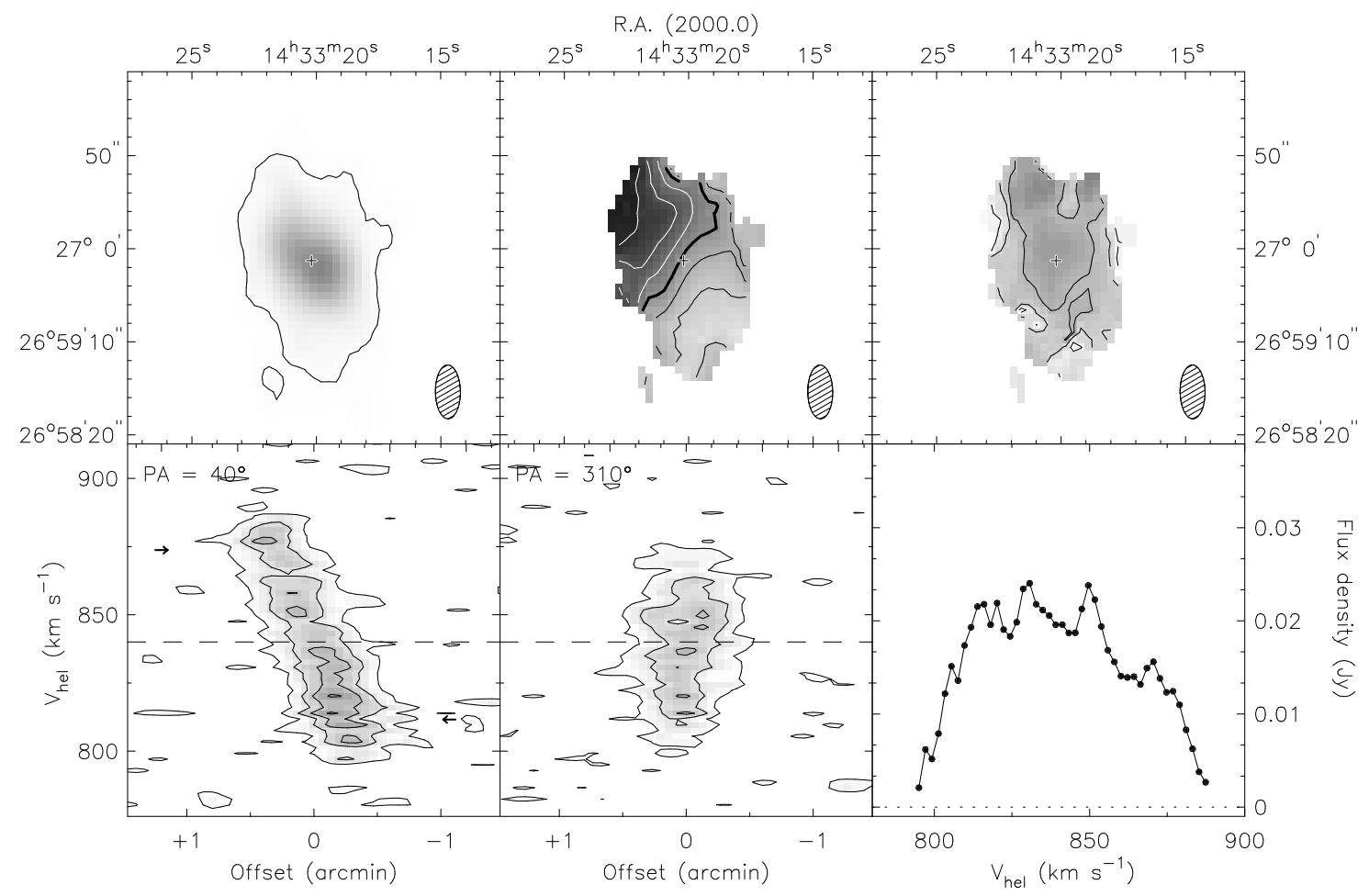

Fig. A.3. Summary panel for D512-2. A general description of the panels and levels is given at the beginning of the Appendix. The $3 \sigma$ contour in the zeroth moment map corresponds to a flux of $5.50 \mathrm{mJy}$, which translates to a column density limit of $n_{\mathrm{HI}}=3.3 \times 10^{19} \mathrm{~cm}^{-2}$.

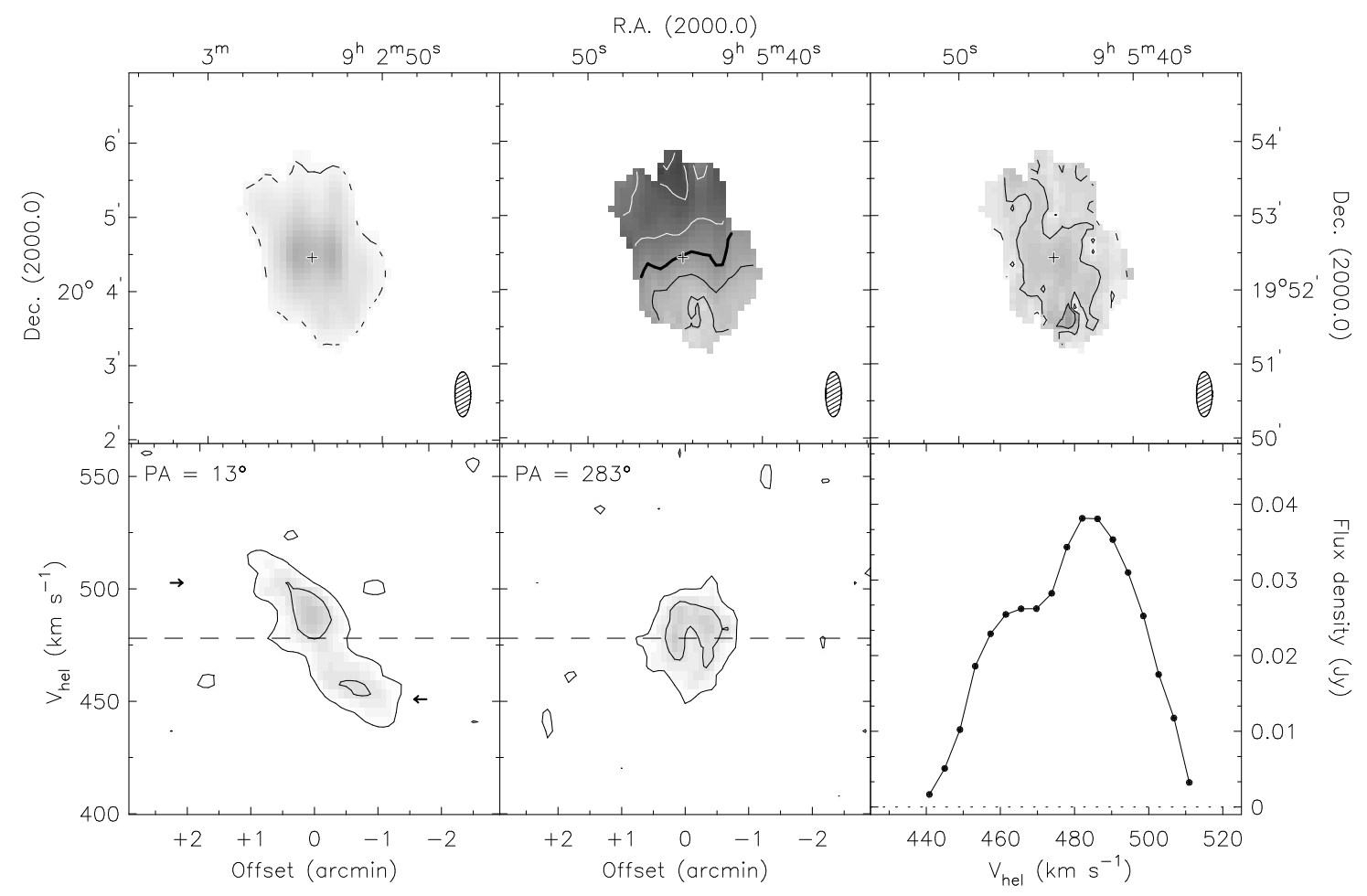

Fig. A.4. Summary panel for D564-8. A general description of the panels and levels is given at the beginning of the Appendix. The $3 \sigma$ contour in the zeroth moment map corresponds to a flux of $4.99 \mathrm{mJy}$, which translates to a column density limit of $n_{\mathrm{HI}}=4.9 \times 10^{19} \mathrm{~cm}^{-2}$. 


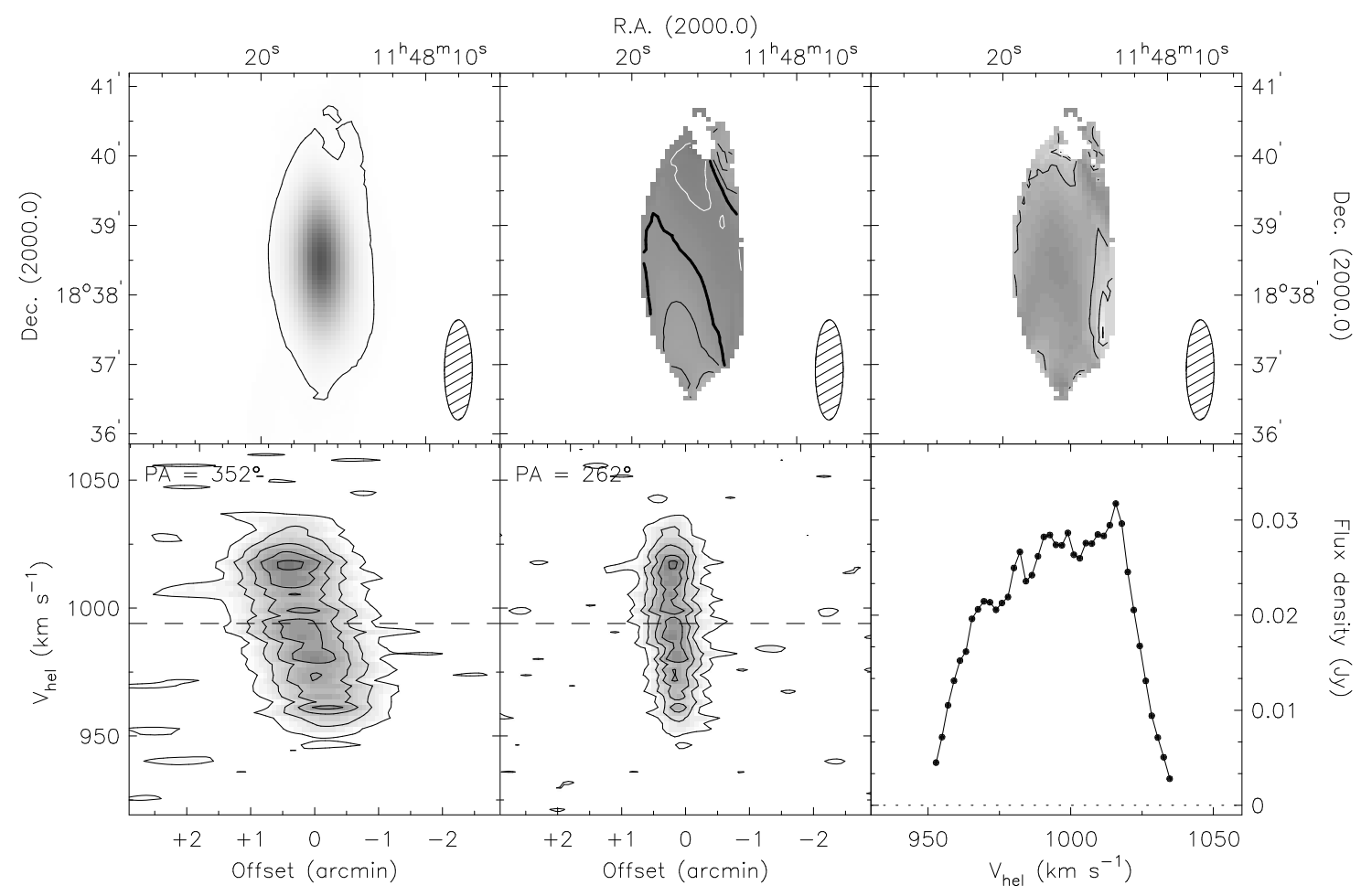

Fig. A.5. Summary panel for D572-5. A general description of the panels and levels is given at the beginning of the Appendix. Grayscales in the zeroth moment map run from a column density of $n_{\mathrm{HI}}=1 \times 10^{19} \mathrm{~cm}^{-2}$ (white) to $n_{\mathrm{HI}}=8 \times 10^{20} \mathrm{~cm}^{-2}$ (black). The $3 \sigma$ contour in the zeroth moment map corresponds to a flux of $12.47 \mathrm{mJy}$, which translates to a column density limit of $n_{\mathrm{HI}}=1.4 \times 10^{19} \mathrm{~cm}^{-2}$.

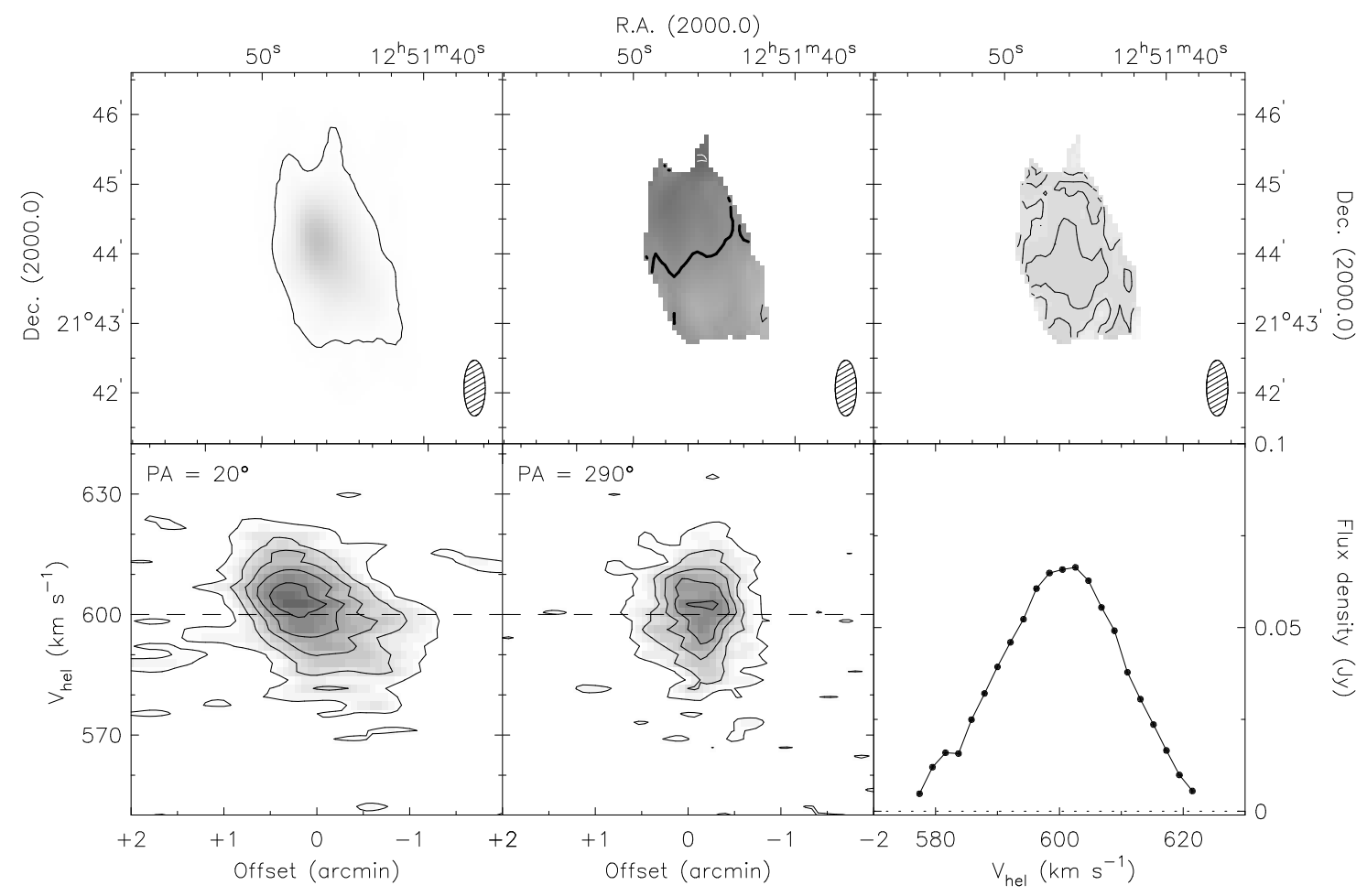

Fig. A.6. Summary panel for D575-1. A general description of the panels and levels is given at the beginning of the Appendix. The $3 \sigma$ contour in the zeroth moment map corresponds to a flux of $9.81 \mathrm{mJy}$, which translates to a column density limit of $n_{\mathrm{HI}}=1.3 \times 10^{19} \mathrm{~cm}^{-2}$. The contours in the second moment map are at $7.5 \mathrm{~km} \mathrm{~s}^{-1}$ and $9 \mathrm{~km} \mathrm{~s}^{-1}$. 


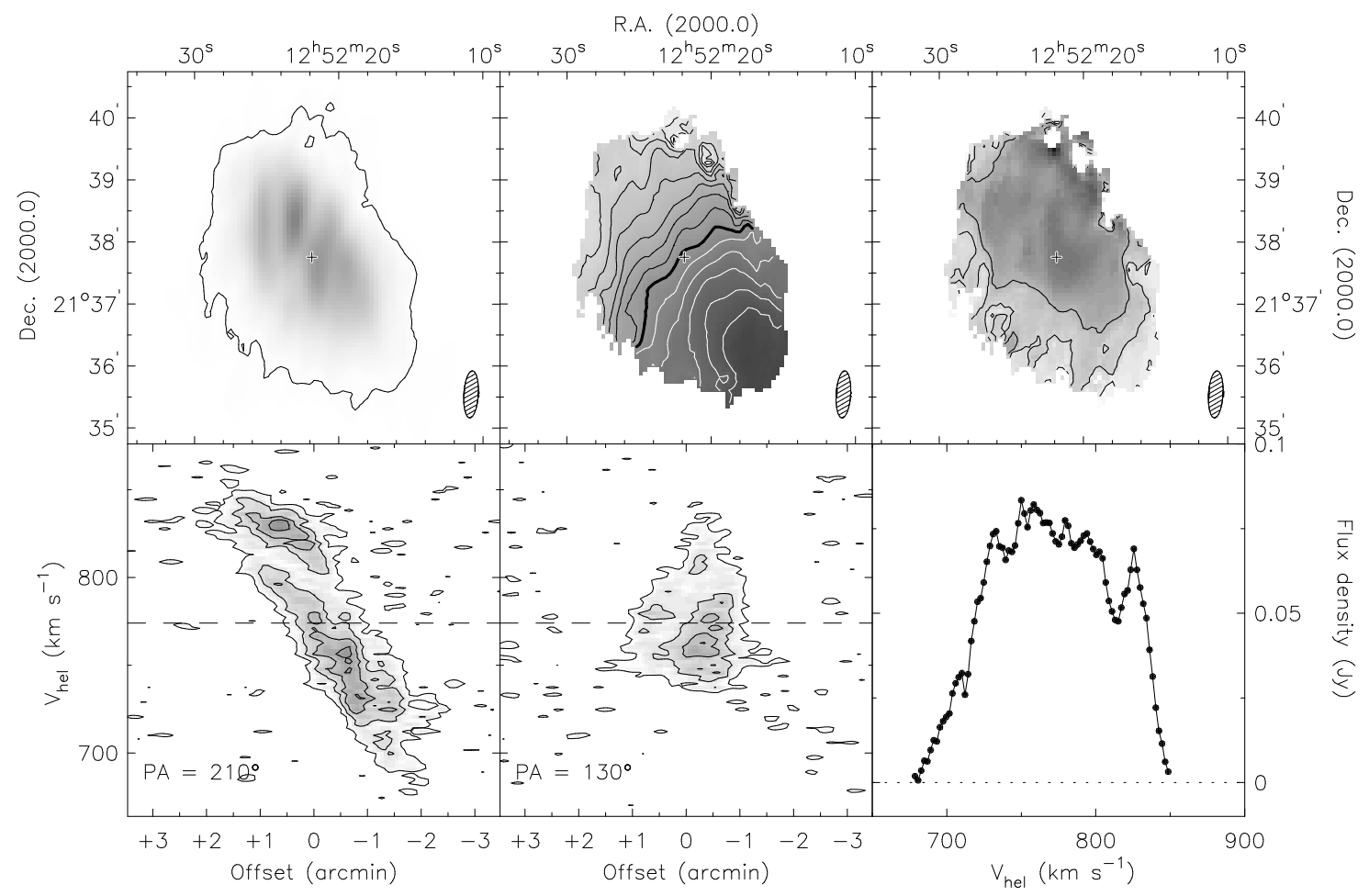

Fig. A.7. Summary panel for D575-2. A general description of the panels and levels is given at the beginning of the Appendix. The $3 \sigma$ contour in the zeroth moment map corresponds to a flux of $5.58 \mathrm{mJy}$, which translates to a column density limit of $n_{\mathrm{HI}}=2.0 \times 10^{19} \mathrm{~cm}^{-2}$.

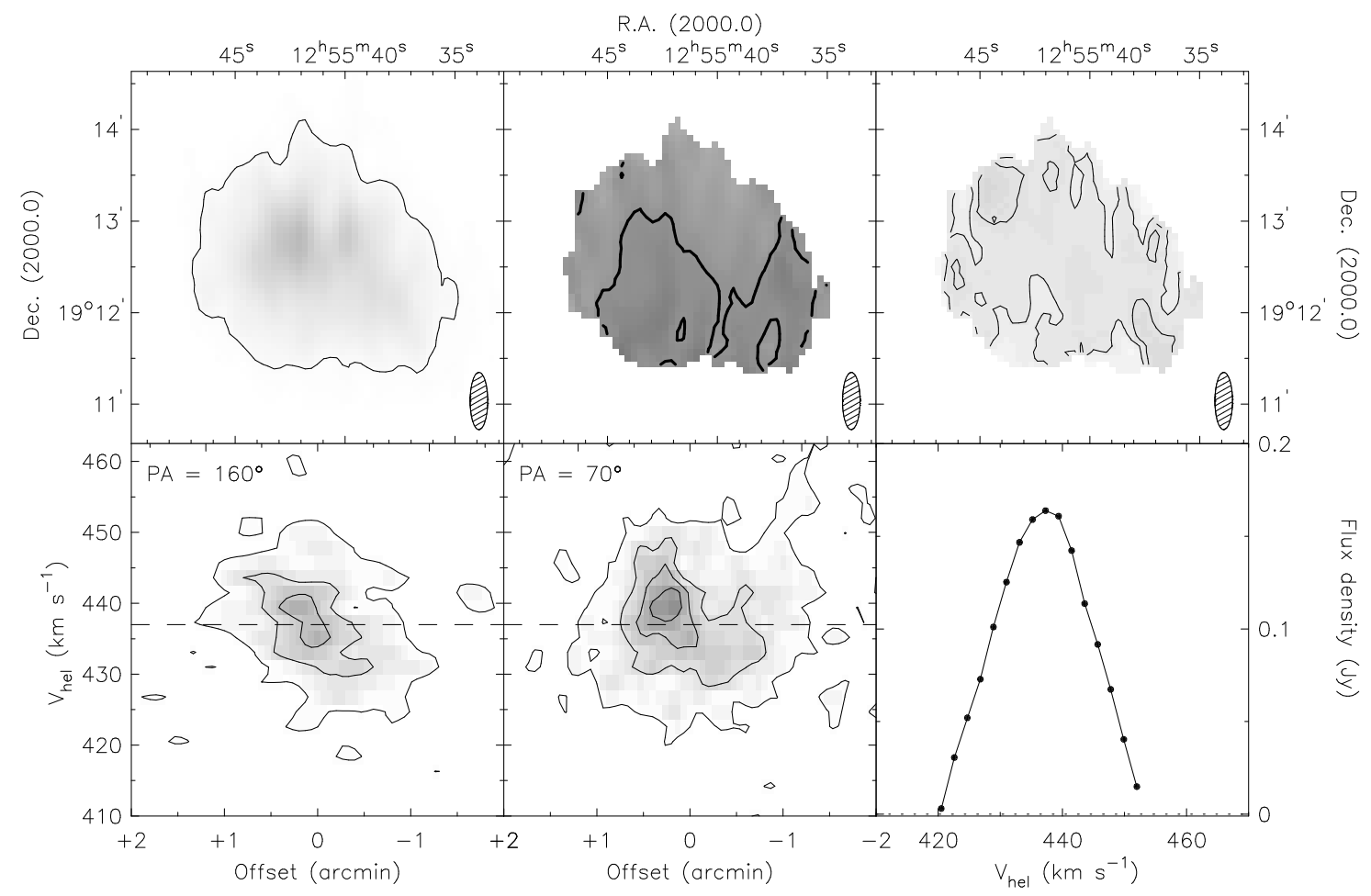

Fig. A.8. Summary panel for D575-5. A general description of the panels and levels is given at the beginning of the Appendix. The $3 \sigma$ contour in the zeroth moment map corresponds to a flux of $8.82 \mathrm{mJy}$, which translates to a column density limit of $n_{\mathrm{HI}}=0.6 \times 10^{19} \mathrm{~cm}^{-2}$. The contours in the second moment map are given at $6 \mathrm{~km} \mathrm{~s}^{-1}$ and $7.5 \mathrm{~km} \mathrm{~s}^{-1}$. 
C. Trachternach et al.: Baryonic Tully-Fisher relation, Online Material p 6

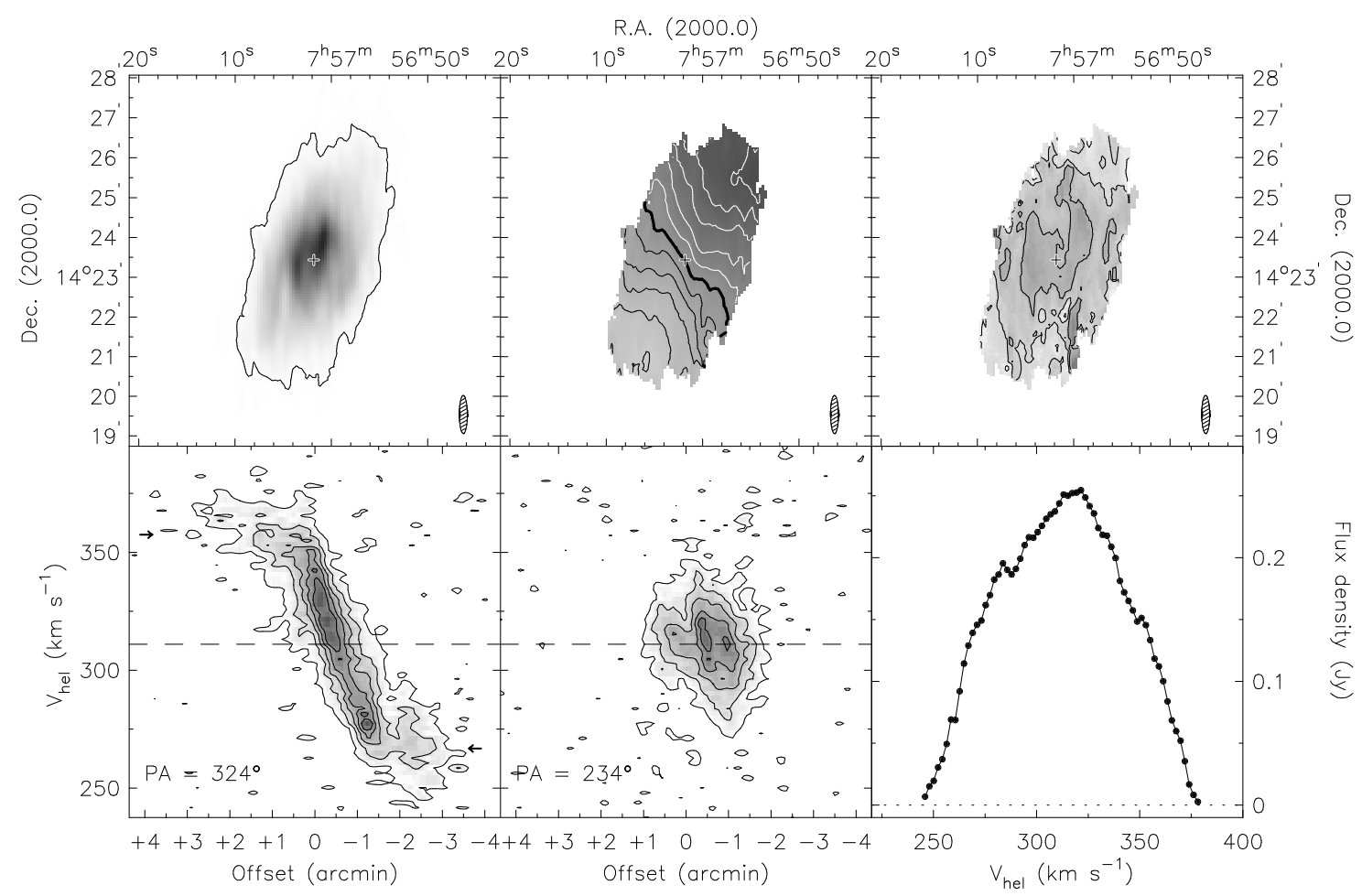

Fig. A.9. Summary panel for D631-7. A general description of the panels and levels is given at the beginning of the Appendix. The $3 \sigma$ contour in the zeroth moment map corresponds to a flux of $11.06 \mathrm{mJy}$, which translates to a column density limit of $n_{\mathrm{HI}}=3.7 \times 10^{19} \mathrm{~cm}^{-2}$.

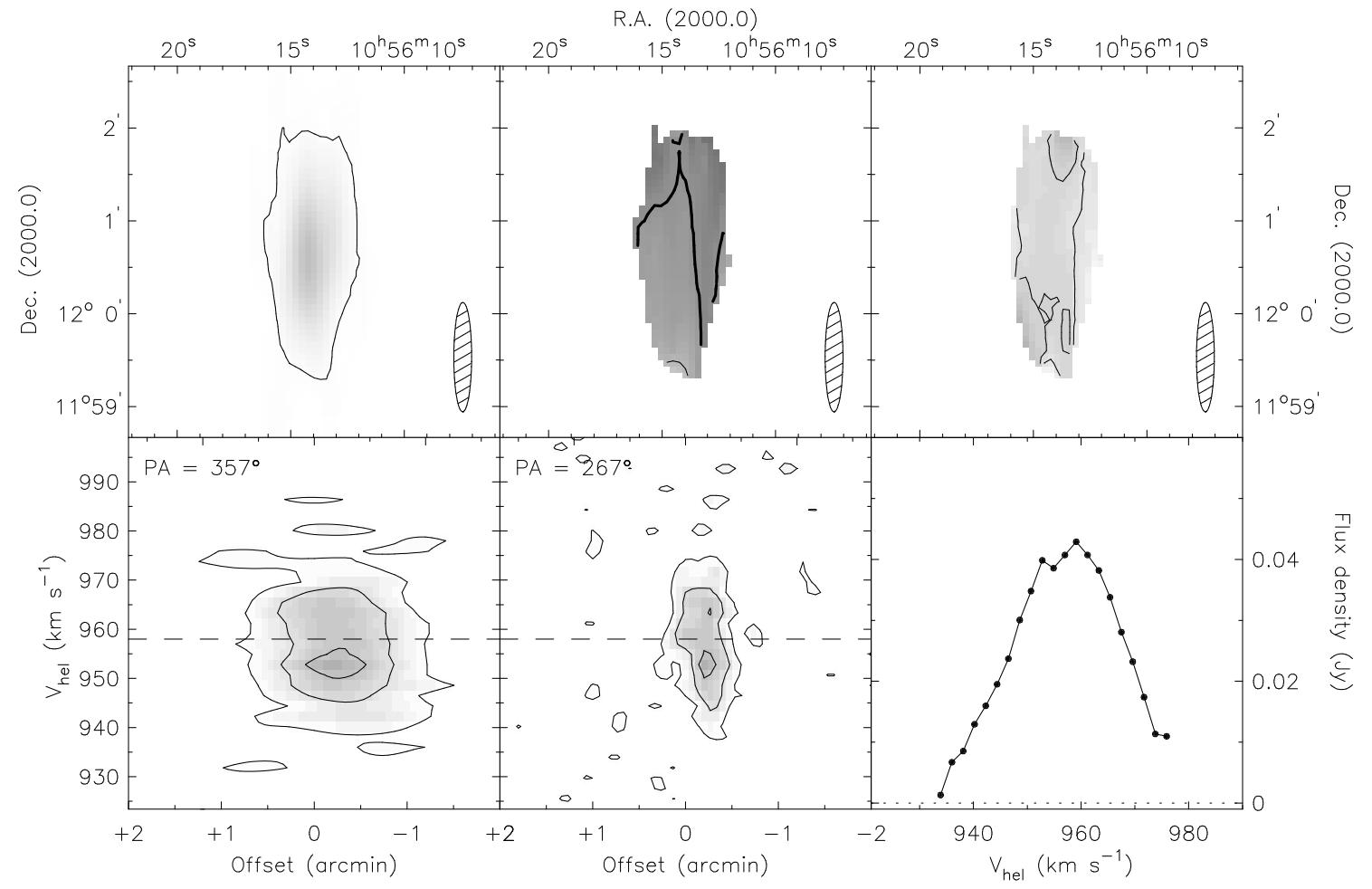

Fig. A.10. Summary panel for D640-13. A general description of the panels and levels is given at the beginning of the Appendix. The $3 \sigma$ contour in the zeroth moment map corresponds to a flux of $11.49 \mathrm{mJy}$, which translates to a column density limit of $n_{\mathrm{HI}}=3.3 \times 10^{19} \mathrm{~cm}^{-2}$. The contours in the second moment map are given at $7.5 \mathrm{~km} \mathrm{~s}^{-1}$ and $10 \mathrm{~km} \mathrm{~s}^{-1}$. 
C. Trachternach et al.: Baryonic Tully-Fisher relation, Online Material p 7

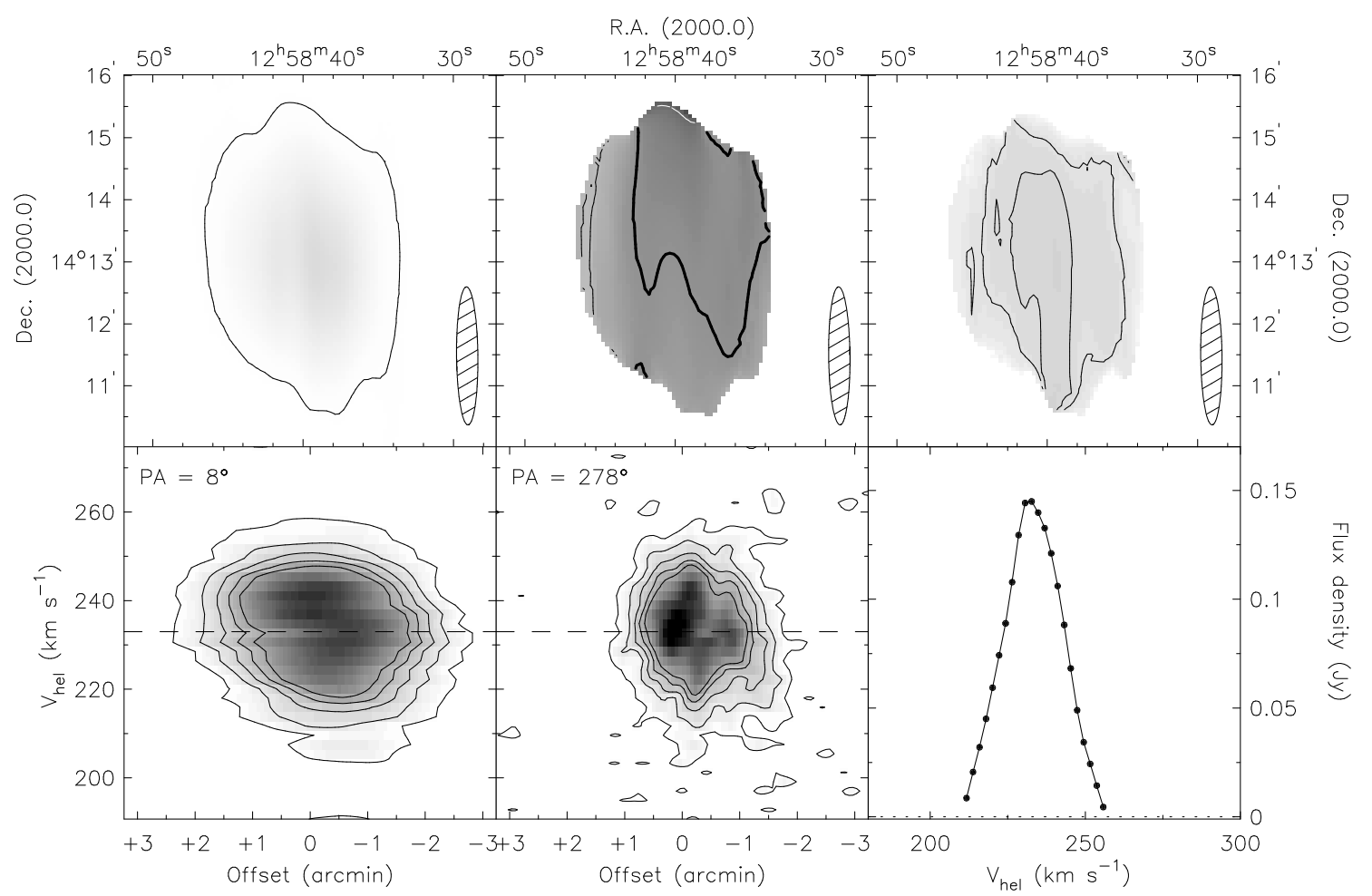

Fig. A.11. Summary panel for D646-7. A general description of the panels and levels is given at the beginning of the Appendix. The $3 \sigma$ contour in the zeroth moment map corresponds to a flux of $7.92 \mathrm{mJy}$, which translates to a column density limit of $n_{\mathrm{HI}}=0.7 \times 10^{19} \mathrm{~cm}^{-2}$. The contours in the second moment map are given at $7.5 \mathrm{~km} \mathrm{~s}^{-1}$ and $9 \mathrm{~km} \mathrm{~s}^{-1}$. 
C. Trachternach et al.: Baryonic Tully-Fisher relation, Online Material p 8

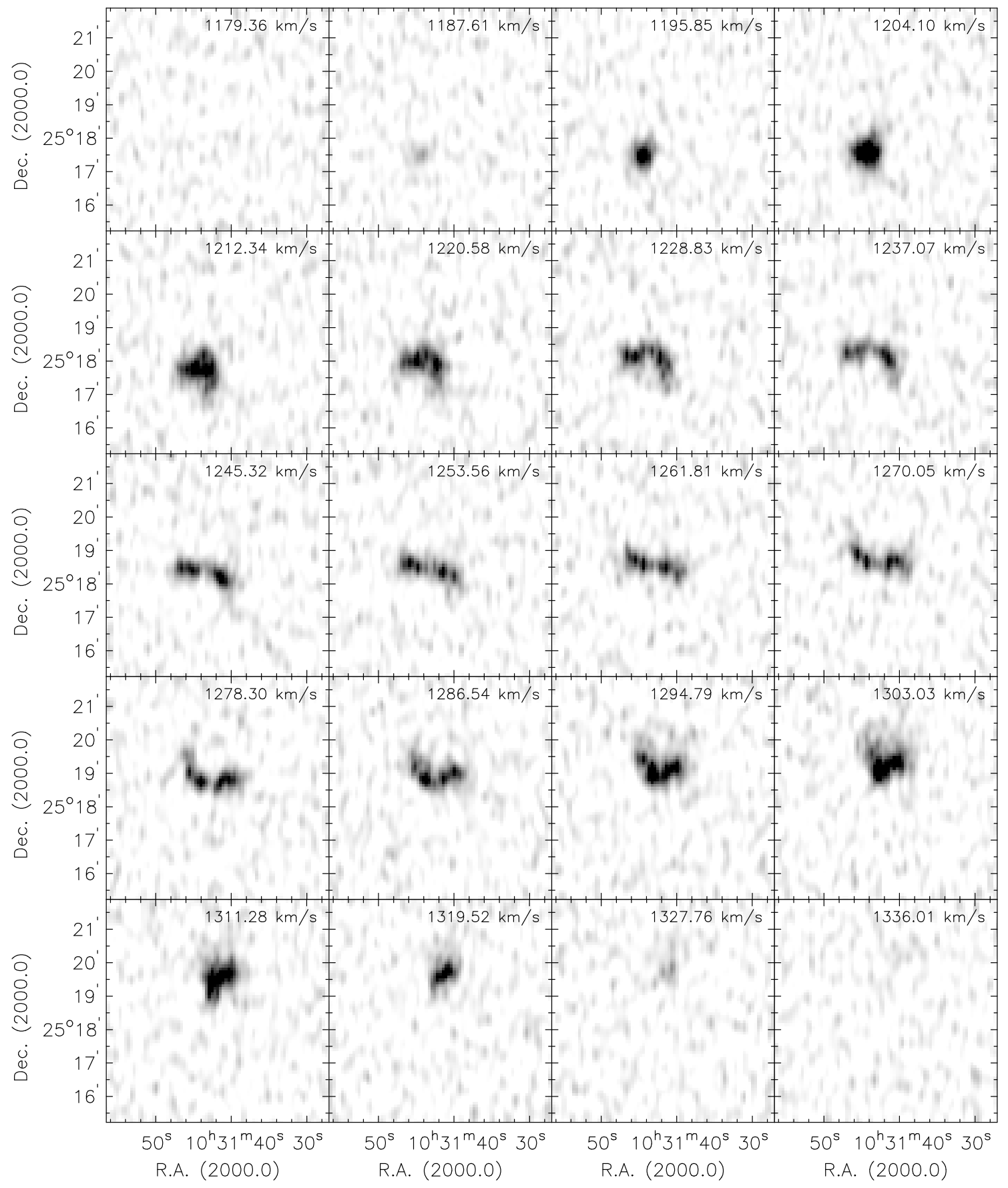

Fig. A.12. Channel maps of D500-2. Grayscales run from -0.02 to $7 \mathrm{mJy}$. Every second channel is shown. 
C. Trachternach et al.: Baryonic Tully-Fisher relation, Online Material p 9

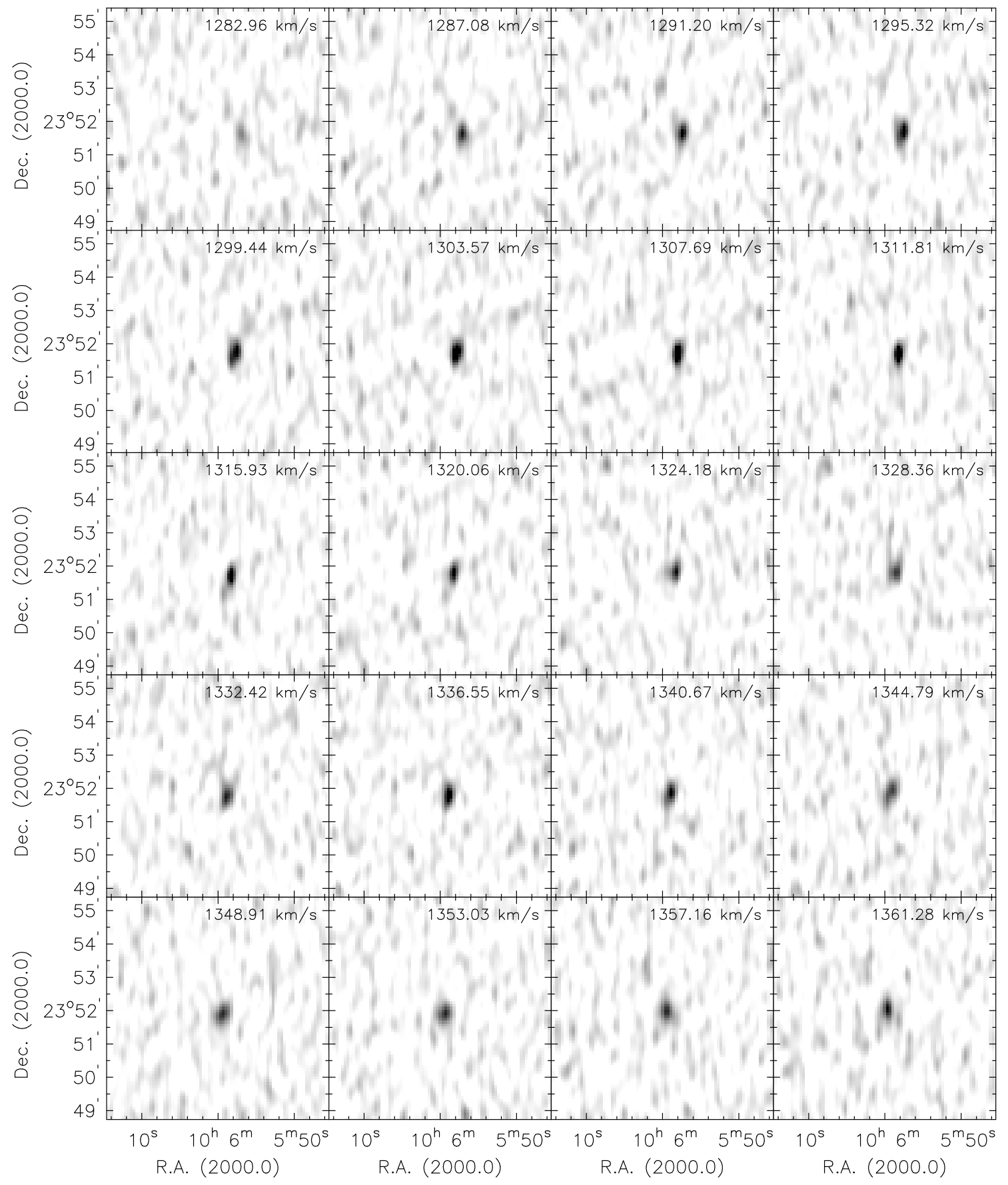

Fig. A.13. Channel maps of D500-3. Grayscales run from -0.02 to $5 \mathrm{mJy}$. Every channel is shown. 
C. Trachternach et al.: Baryonic Tully-Fisher relation, Online Material p 10

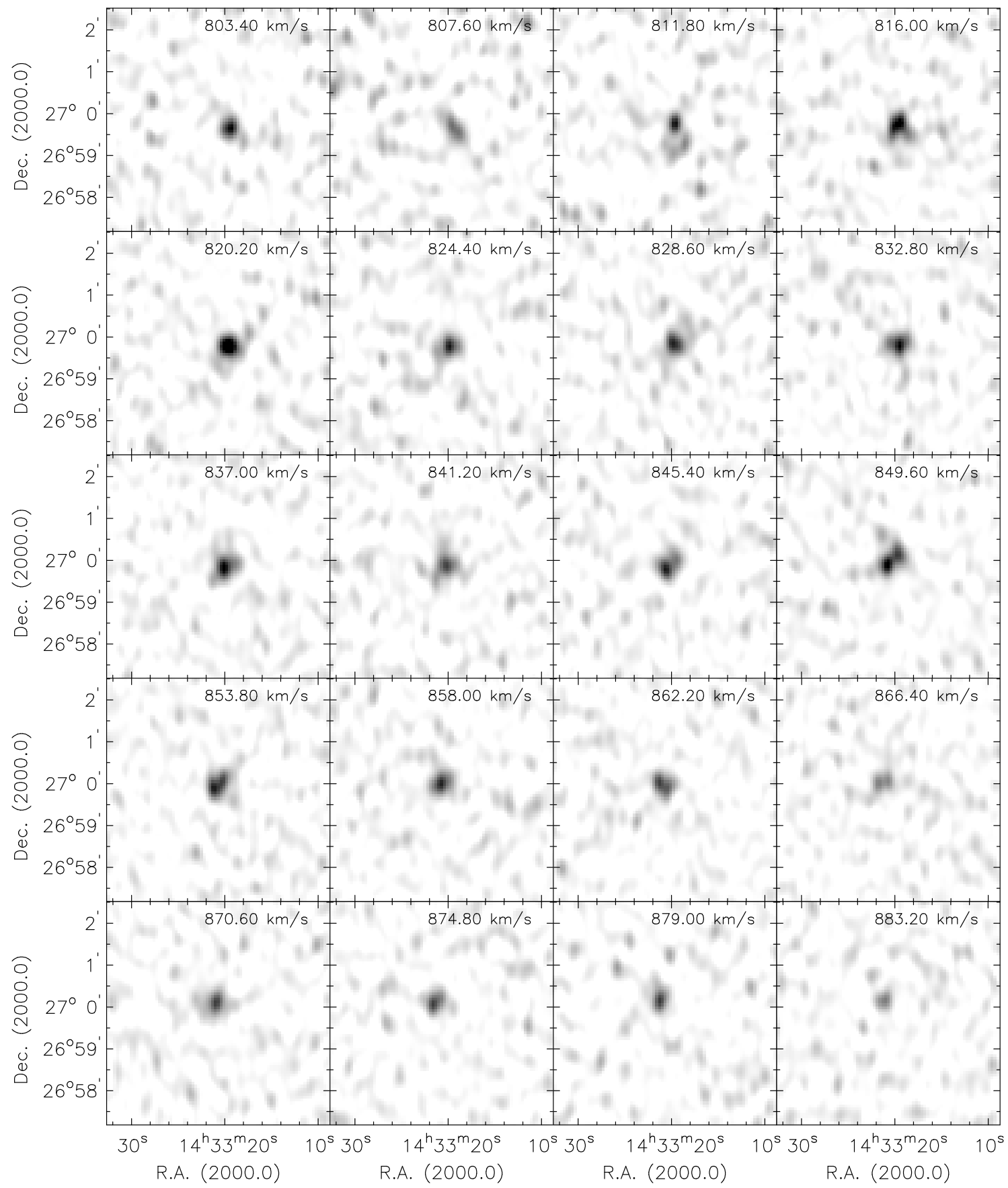

Fig. A.14. Channel maps of D512-2. Grayscales run from -0.02 to 8 mJy. Every second channel is shown. 
C. Trachternach et al.: Baryonic Tully-Fisher relation, Online Material p 11

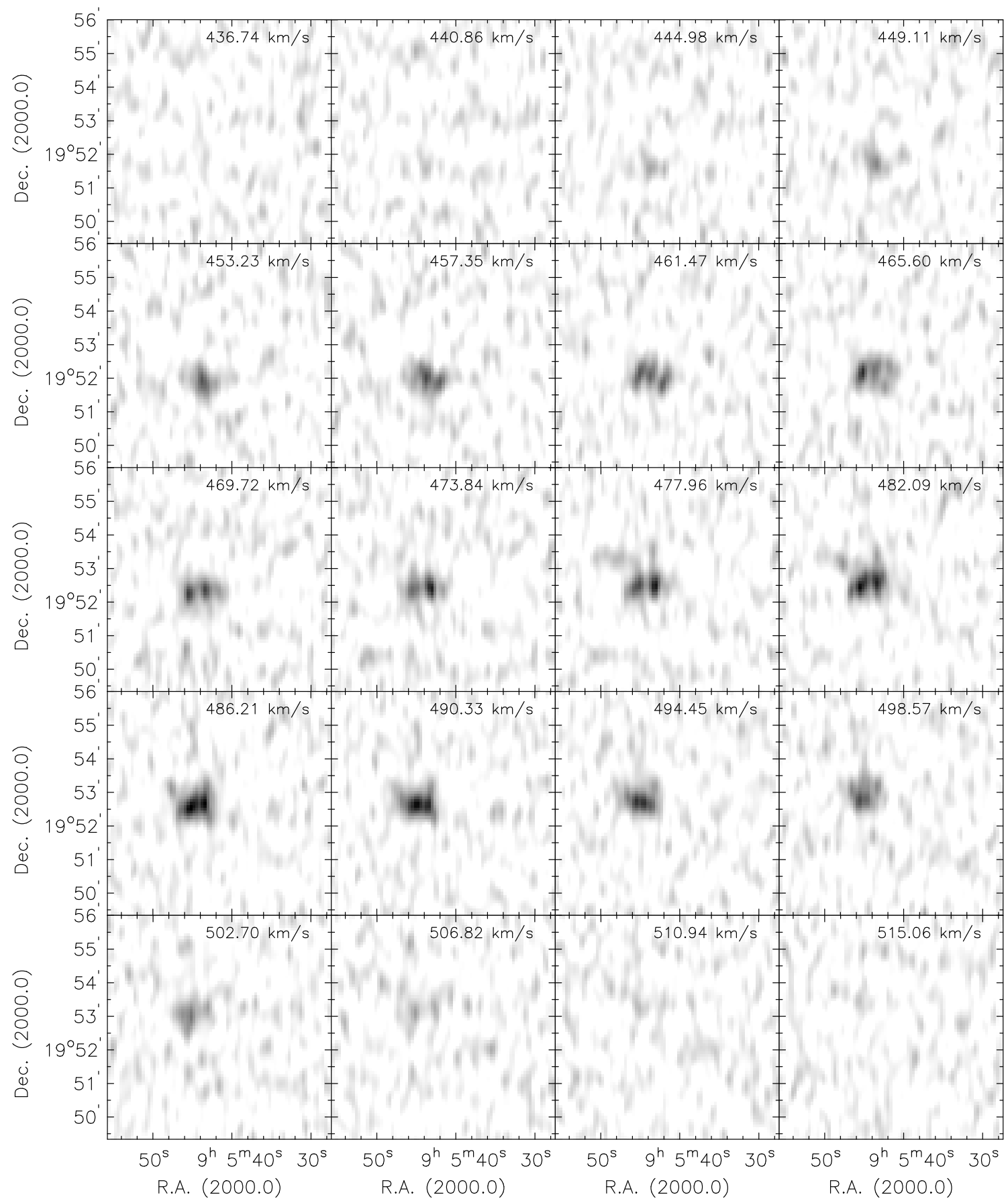

Fig. A.15. Channel maps of D564-8. Grayscales run from -0.02 to $7 \mathrm{mJy}$. Every channel is shown. 
C. Trachternach et al.: Baryonic Tully-Fisher relation, Online Material p 12

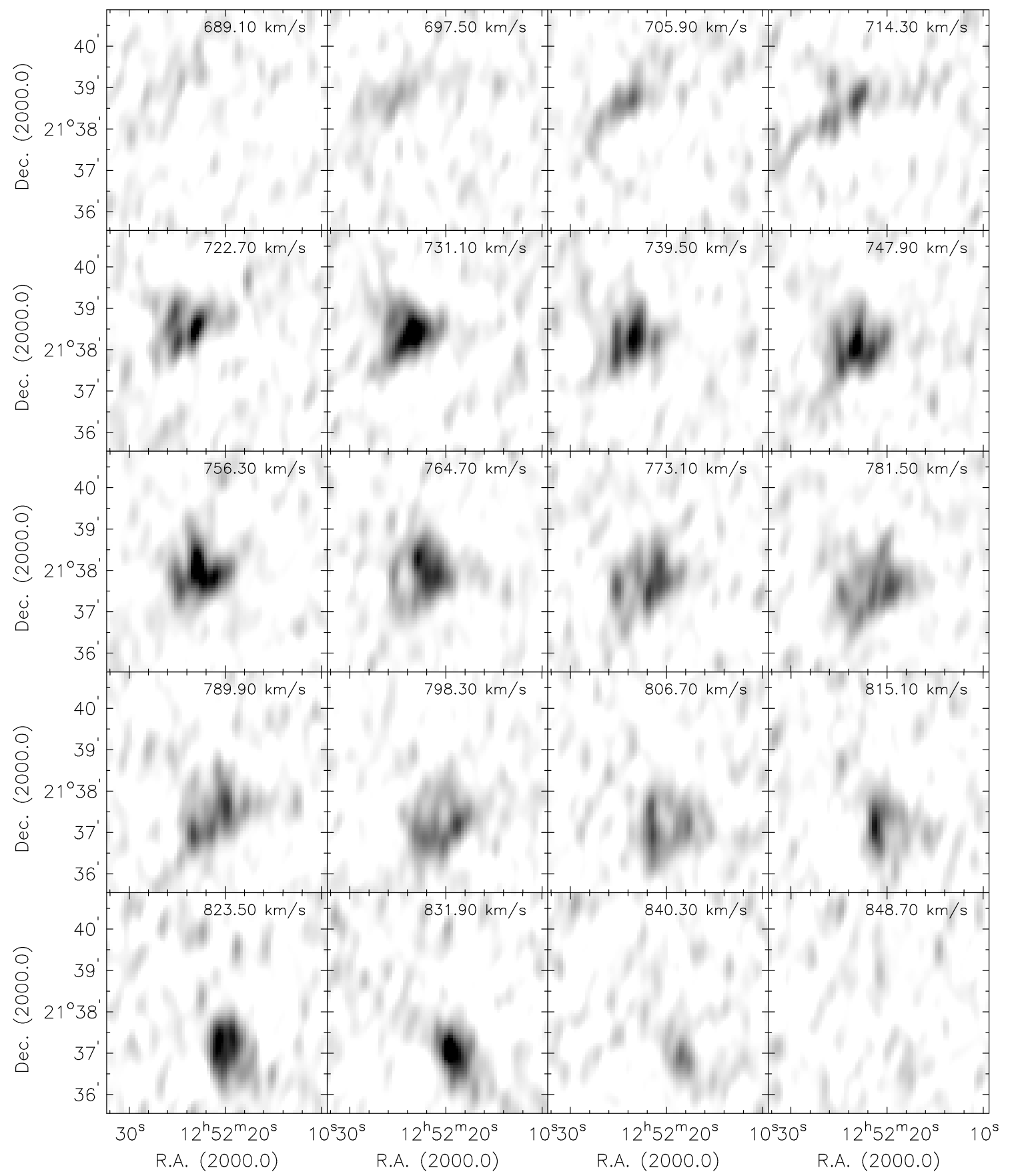

Fig. A.16. Channel maps of D575-2. Grayscales run from -0.02 to $10 \mathrm{mJy}$. Every fourth channel is shown. 
C. Trachternach et al.: Baryonic Tully-Fisher relation, Online Material p 13

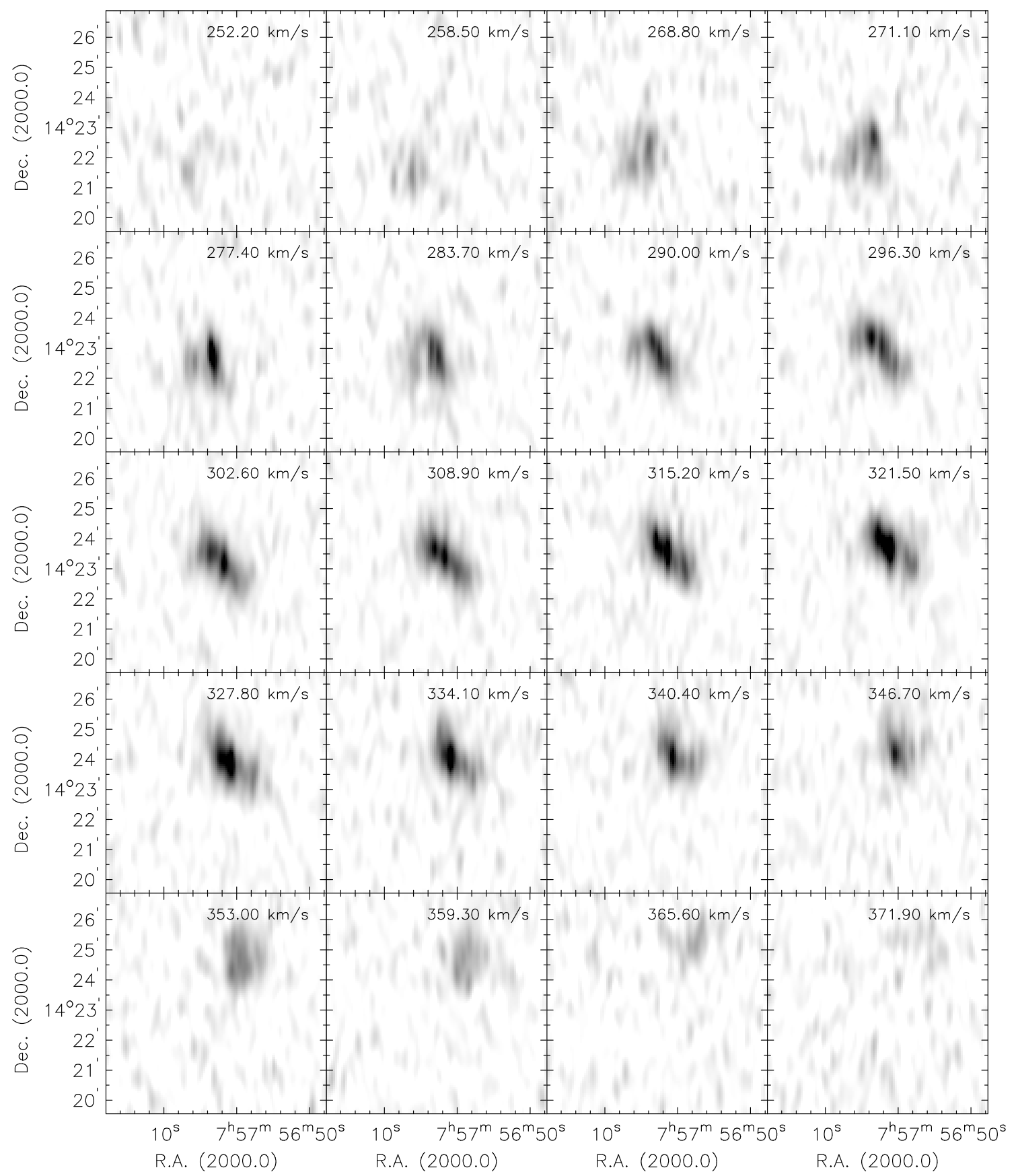

Fig. A.17. Channel maps of D631-7. Grayscales run from -0.02 to $25 \mathrm{mJy}$. Every second channel is shown. 